\title{
Accelerating Membrane Simulations with Hydrogen Mass Repartitioning
}

By: Curtis Balusek, Hyea Hwang, Chun Hon Lau, Karl Lundquist, Anthony Hazel, Anna Pavlova, Diane L. Lynch, Patricia H. Reggio, Yi Wang, and James C. Gumbart

Balusek, C., Hwang, H., Lau, C.H., Lundquist, K., Hazel, A., Pavlova, A., Lynch, D.L., Reggio, P.H., Wang, Y., \& Gumbart, J.C. Accelerating Membrane Simulations with Hydrogen Mass Repartitioning. Journal of Chemical Theory and Computation 2019, 15, 8, 4673-4686. https://doi.org/10.1021/acs.jctc. $9 \mathrm{~b} 00160$

This document is the Accepted Manuscript version of a Published Work that appeared in final form in Journal of Chemical Theory and Computation, copyright (C) American Chemical Society after peer review and technical editing by the publisher. To access the final edited and published work see https://doi.org/10.1021/acs.jctc.9b00160.

Abstract:

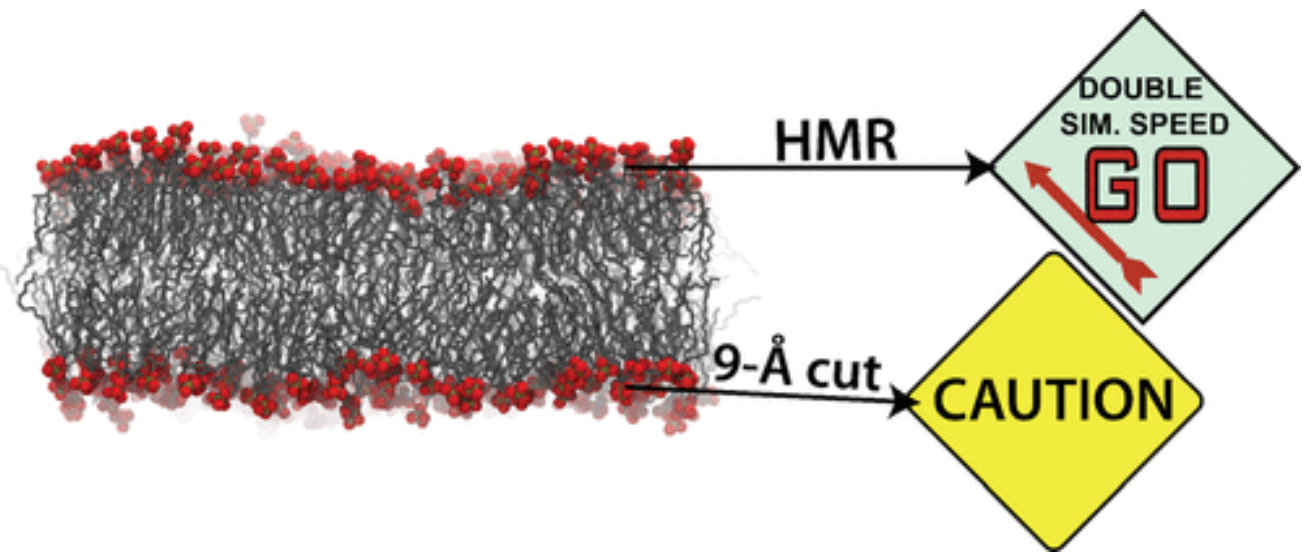

The time step of atomistic molecular dynamics (MD) simulations is determined by the fastest motions in the system and is typically limited to $2 \mathrm{fs}$. An increasingly popular approach is to increase the mass of the hydrogen atoms to $\sim 3 \mathrm{amu}$ and decrease the mass of the parent atom by an equivalent amount. This approach, known as hydrogen-mass repartitioning (HMR), permits time steps up to $4 \mathrm{fs}$ with reasonable simulation stability. While HMR has been applied in many published studies to date, it has not been extensively tested for membrane-containing systems. Here, we compare the results of simulations of a variety of membranes and membrane-protein systems run using a 2 fs time step and a 4 fs time step with HMR. For pure membrane systems, we find almost no difference in structural properties, such as area-per-lipid, electron density profiles, and order parameters, although there are differences in kinetic properties such as the diffusion constant. Conductance through a porin in an applied field, partitioning of a small peptide, hydrogen-bond dynamics, and membrane mixing show very little dependence on HMR and the time step. We also tested a $9 \AA$ cutoff as compared to the standard CHARMM cutoff of $12 \AA$, finding significant deviations in many properties tested. We conclude that HMR is a valid approach for membrane systems, but a $9 \AA$ cutoff is not.

Keywords: lipids | molecular dynamics | diffusion membranes 


\section{Article:}

\section{Introduction}

Biological membranes are an essential component of all living cells. ${ }^{(1)}$ They serve as a barrier between the cell and the outside world, preventing entry of many potentially harmful compounds, as well as regulating cellular import and export through membrane proteins. Cell membranes are typically composed of a phospholipid bilayer with embedded and/or associated proteins. ${ }^{(1)}$ Phospholipids are amphiphilic molecules that are characterized by a hydrophilic headgroup containing a phosphate, which is glycerol-linked to one or more hydrophobic fattyacid tails (Figure 1A). ${ }^{(1)}$

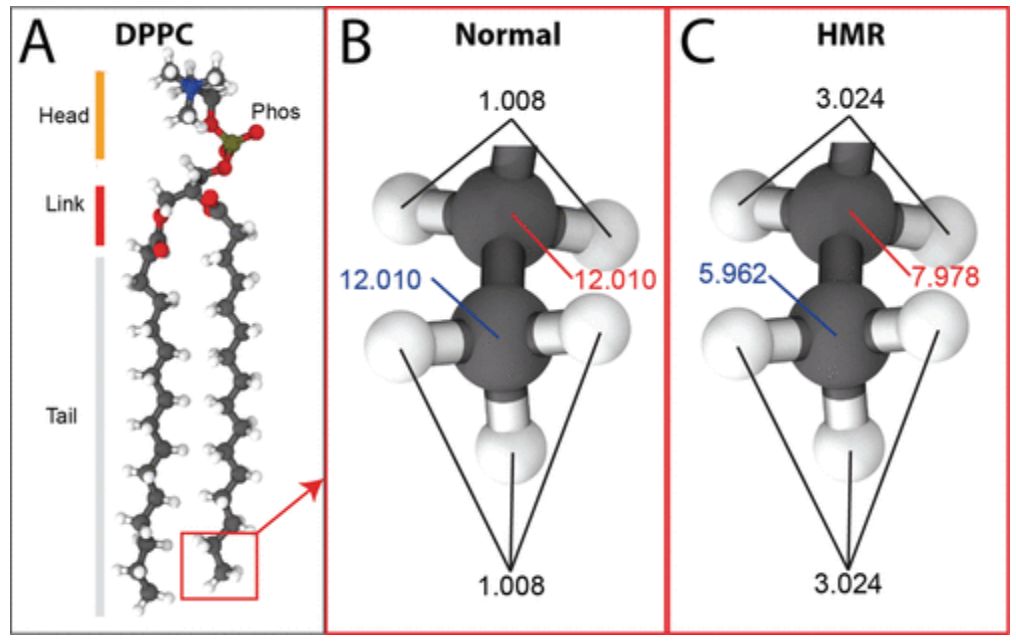

Figure 1. (A) Structure of DPPC lipid. Carbons are colored in dark gray, hydrogens in white, oxygens in red, nitrogen in blue, and phosphorus in tan. "phos" and "link" denote phosphate and ester linkages, respectively. (B) Normal mass distribution for the atoms in the acyl-chain tail. (C) Mass distribution for the same atoms with HMR.

Membrane models are frequently used in molecular dynamics (MD) studies because of their biological relevance. As such, MD simulations can be used to study membrane properties and provide an atomistic description of membrane structure and dynamics. ${ }^{(2-8)}$ Additionally, membrane permeability and small-molecule interactions are often of interest in drug design, which can be investigated computationally using membrane models. ${ }^{(9,10)}$

A major challenge in membrane simulations is the need for accurate lipid force fields. ${ }^{(11,12)}$ To date, multiple force fields have been developed: AMBER14, ${ }^{(13)}$ SLIPIDS, ${ }^{(14)}$ CHARMM36, ${ }^{(15)}$ and multiple GROMACS united-atom models; ${ }^{(16-18)}$ several studies have compared these force fields and improved upon existing ones. ${ }^{(2,9,11,12,18)}$ Force-field parameters are typically evaluated on the basis of their ability to reproduce experimentally known structural and dynamic properties of pure-lipid bilayers, for example, lipid area, bilayer thickness, compressibility modulus, deuterium order parameters, and diffusion coefficients. ${ }^{(2,8,11,12)}$ In some cases, partition coefficients have been calculated to validate lipid:small-molecule interactions. ${ }^{(7)}$ Furthermore, 
compatibility with water and protein force fields should be considered when choosing a lipid force field. ${ }^{(19,20)}$

The CHARMM36 (C36) lipid force field is frequently used in MD simulations because it can accurately reproduce a number of physical properties of lipids, as well as its compatibility with the C36 protein and CGenFF small-molecule force field. ${ }^{(2,7,8,12,15,19,20)}$ The most recent C36 lipid force field update resulted in improved agreement with experimental order parameters, compressibility modulus, and area per lipid. ${ }^{(15)}$ Furthermore, the recently launched CHARMMGUI web interface, which supports several MD software packages, has greatly facilitated the construction of membrane systems for MD simulations, specifically utilizing the $\mathrm{C} 36$ force field. ${ }^{(21-24)}$ CHARMM-GUI automatically generates structures, coordinates, parameters, and input files for pure membrane and membrane-protein systems, supporting numerous phospholipid molecules.

To study properties of a membrane system, multiple simulations of sufficiently long time scales are typically required. ${ }^{(9,25)}$ As such, there is significant benefit to improving simulation efficiency, particularly for membrane-containing systems, which can be much larger than protein-only systems. Previously suggested approaches to speed up MD simulations include using a longer time step. ${ }^{(26,27)}$ Implementing a longer time step decreases the accuracy when integrating the equations of motion in MD; however, it has been shown that the introduced errors are typically much smaller than the statistical errors due to limited sampling. ${ }^{(28)}$ Additionally, the increased energy drift introduced by a longer time step can be dampened by using a thermostat. ${ }^{(27,28)}$ Currently, the magnitude of the time step in atomistic MD is limited by the fastest-moving atoms in the simulation, which are the vibrational motions of the hydrogen atoms. ${ }^{(26)}$ Therefore, increases in time step can be achieved by slowing or restricting the movement of the hydrogens. ${ }^{(26)}$ Common practice in MD simulation has been an increased time step from 1 to 2 fs by keeping the covalent bonds involving hydrogen atoms rigid using SETTLE and SHAKE algorithms for water and other molecules, respectively. ${ }^{(29,30)}$ The implementation of these algorithms nearly doubles the achievable simulation time at fixed computational cost; however, the SHAKE algorithm is likely to fail for time steps beyond 2 fs for conventional MD. ${ }^{(27,29)}$

Recently, MD simulation studies have shown that time steps of up to 4 or 5 fs can be achieved by altering hydrogen masses. ${ }^{(26,27,31)}$ It is important to note that when implementing mass modifications, it is requisite that the total mass of the system does not change. ${ }^{(26,27)}$ As described by Feenstra et al., increasing the total mass of the system will result in a slower time scale for various events of interest, for example, diffusion. ${ }^{(26)}$ Similarly, in the virtual site technique (VST), the hydrogens' masses are assigned to the adjacent heavy atoms, and their positions are calculated and updated on the basis of the positions of the heavy atoms. ${ }^{(31,32)}$ However, implementing VST requires reoptimization of force field parameters, such that when applied to the C36 force field, VST was shown to alter several lipid properties, leading to thinner and more disordered bilayers. ${ }^{(31)}$ Recently, it was found that the combination of VST with HMR on every fourth methyl group in the lipid tails resulted in excellent agreement with measured lipid properties in standard MD simulations. ${ }^{(33)}$ 
To clarify, HMR modifies the atomic input by repartitioning mass from each heavy atom to its covalently bonded hydrogens, while conserving the overall molecular mass. ${ }^{(26,27)}$ Thus, when applying HMR, the reweighted hydrogen mass should not be greater than 3 amu because a larger mass transfer would make methyl carbons lighter than their bonded hydrogens. While in theory, such an increase in hydrogen mass only allows the time step to be increased by approximately a factor of $\sqrt{3}$, a 4 fs time step has routinely been used. For instance, Hopkins et al. illustrated this method for both a small peptide as well as a large protein in explicit solvent. ${ }^{(27)}$ They found that the protein/peptide with HMR applied, using a hydrogen mass of 3 amu and a time step of $4 \mathrm{fs}$, consistently reproduced conformations observed without HMR. However, when HMR was applied to both protein and water molecules, there was an increase in the viscosity of water and, consequently, slower transition rates between different protein conformations. Therefore, HMR should not be applied to water. Since its inception, HMR has been used in several software packages such as NAMD,${ }^{(34)}$ AMBER, ${ }^{(35)}$ and ACEMD,${ }^{(36)}$ to speed up MD simulation output.

It has also been shown that additional speed up can be obtained by decreasing the cutoff for nonbonded interactions. Although C36 lipids were parametrized and validated using a $12 \AA$ cutoff with a force-based switching function applied at $8 \AA,{ }^{(15)}$ most HMR studies employing lipids to date have used the C36 lipid force field with a $9 \AA$ cutoff (default setting) in ACEMD or AMBER. ${ }^{(37-39)}$ Previous MD simulations have shown that membrane properties are highly sensitive to the cutoff value and Lennard-Jones (LJ) switching functions because lipid dynamics are primarily driven by LJ interactions. ${ }^{(15,24)}$ Additionally, lipids are more hydrogen rich than proteins, for which HMR was previously validated. ${ }^{(27)}$ However, to date, the effects of HMR and a shorter $9 \AA$ cutoff with the C36 lipid force field have not been systematically investigated.

In this Article, we test the application of HMR with a 4 fs time step to membrane systems by comparing membrane properties and lipid-protein interactions. Additionally, the effects of $9 \AA$ cutoff are examined. Several single-lipid, mixed-lipid, and protein-embedded membrane systems were studied. It is found that applying HMR with a $12 \AA$ cutoff provides consistent results in comparison to the conventional $2 \mathrm{fs}$ time step and $12 \AA$ cutoff MD across all studied systems. However, employing a $9 \AA$ cutoff altered several structural and kinetic properties for lipid bilayers, as well as protein dynamics in some, but not all, cases.

\section{Methods}

Construction and Analysis of Pure Membrane Systems

All-atom lipid bilayers were generated for pure membrane systems using CHARMM-GUI. ${ }^{(23)}$ Three pure membrane models (DPPC, POPE, and DOPC) were generated, as well as a fourth, multiple-lipid "Top6" model. ${ }^{(40)}$ Each system contained 480 lipids (240/leaflet) and was solvated and ionized to a concentration of $150 \mathrm{mM} \mathrm{NaCl}$. Although smaller (60 lipids/leaflet) membranes were attempted initially, it was found that many of the properties measured either did not converge well in $100 \mathrm{~ns}$ simulations or disagreed with previously reported simulation results (data not shown). Further details about each system and constitutive components can be found in Table S1. Upon completion of the simulations, trajectories were analyzed to measure structural and kinetic properties such as area per lipid (APL), membrane thickness, deuterium 
order parameters, electron density profile, compressibility modulus $\left(K_{\mathrm{A}}\right)$, diffusion coefficient, dihedral trans-gauche transition rates, and hydrogen-bond lifetimes.

In the present work, the APL for all lipid membranes was computed from the area of the simulation box in the $x-y$ plane divided by the number of lipids in each leaflet (240). Because we employed anisotropic pressure coupling, the simulation box was allowed to fluctuate during the simulation; APL was used to monitor simulation equilibrium. Membrane thickness is reported as the head-to-head average distance as measured in the electron density profiles, which was calculated using a simple binning procedure, as opposed to more precise methods designed for larger membranes. ${ }^{(41)} K_{\mathrm{A}}$ is a measure of the stiffness of the membrane and was calculated as

$$
K_{\mathrm{A}}=k_{\mathrm{B}} T\left(\frac{A}{\sigma_{\mathrm{A}}^{2}}\right)
$$

where $k_{\mathrm{B}}$ is Boltzmann's constant, $T$ is the temperature, $A$ is the area, and $\sigma_{\mathrm{A}}{ }^{2}$ is the variance of the area during the simulation.

Diffusion values $(D)$ are measured from the mean-squared displacement of lipids in the $x-y$ plane according to

$$
\left\langle\Delta r(\tau)^{2}\right\rangle=\frac{1}{N} \sum_{i=1}^{N}\left\langle|| \vec{r}_{i}(t+\tau)-\vec{r}_{i}(t)||^{2}\right\rangle=4 D \tau
$$

The sum occurs over all lipids and is averaged over all time separations $\tau$. The first $1 \mathrm{~ns}$ of time lag is discarded. The diffusion coefficient is then obtained from the slope of the linear regime of mean-squared displacement versus time-separation. All diffusion values are measured over the last $50 \mathrm{~ns}$ of simulation with $\tau$ up to $20 \mathrm{~ns}$. Any center-of-mass drift of each monolayer was removed prior to calculating the mean-squared displacement.

Deuterium order parameters $\left(S_{\mathrm{CD}}\right)$ are used to compare lipid simulations to experimental results of membrane systems for each acyl carbon in the aliphatic tail, which are given by

$$
S_{\mathrm{CD}}=\left\langle\frac{1}{2}\left(3 \cos ^{2} \theta-1\right)\right\rangle
$$

where $\theta$ is the angle measured between the carbon-hydrogen bond vector and the membrane normal. The pure membrane systems simulated here are small enough to not exhibit large-scale undulations, such that the normal vector to a membrane can be assumed to be parallel to the $z$ axis.

Construction and Analysis of Mixed Membrane and Large POPC Systems

System construction and Anton simulation details of the mixed POPC:cholesterol membrane (POPC:CHL, see Table S1) were described in Hong et al. ${ }^{(42)}$ A POPC bilayer with 680 lipids was constructed by replicating an equilibrated bilayer with 170 POPC lipids four times. ${ }^{(42)}$ All 
simulation conditions of this large POPC bilayer as well as HMR simulations using a 4 fs time step and 12 or $9 \AA$ cutoff of the mixed POPC:CHL membrane were identical to those of the pure lipid bilayers described below.

Radial pair distribution function and clustering analysis of the mixed POPC:CHL membrane were performed following Hong et al. ${ }^{(42)}$ Undulation analysis of the 680 -lipid POPC bilayer was performed using the MDAnalysis package. ${ }^{(43)}$ Error estimation of the bending modulus $k_{\mathrm{c}}$ was performed as the following: A simulation trajectory was divided into $M$ blocks, each of length $\tau_{\mathrm{b}}$. The average of $u^{2}(q)$, the square amplitude of undulation at a given wavenumber $q$, from each block was determined and then used to compute a standard deviation $\sigma_{\tau \mathrm{v}}$, on the basis of which we obtained the blocked standard error (BSE): ${ }^{(44)}$

$$
\mathrm{BSE}=\frac{\sigma_{\tau_{\mathrm{b}}}}{\sqrt{M}}
$$

The error in $k_{\mathrm{c}}$ was then determined by assuming a "worst-case-scenario combination" of errors from the four wavenumbers analyzed here: we subtracted the BSE from $\left\langle u^{2}(q)\right\rangle$ for the lowest wavenumber and added the corresponding the BSEs to $\left\langle u^{2}(q)\right\rangle$ for the remaining three wavenumbers, followed by refitting of a first-order polynomial. This procedure resulted in the upper-bound error in $k_{\mathrm{c}}$. Conversely, a lower-bound error was obtained by adding the BSE to $\left\langle u^{2}(q)\right\rangle$ for the lowest wavenumber and subtracting it from $\left\langle u^{2}(q)\right\rangle$ for the remaining three wavenumbers. We note that the thus-obtained errors were asymmetric.

Applied Electric Field Simulations

An OmpF membrane-protein system was created using the crystal structure reported by Yamashita et al. ${ }^{(45)}$ (PDB: 2ZFG) and embedded in a POPE phospholipid bilayer to replicate the systems used in Pezeshki et al. ${ }^{(46)}$ The CHARMM36 protein force field was used. ${ }^{(20)}$ The system contained 99157 atoms with 176 POPE lipids, 19421 water molecules, and 420 potassium $\left(\mathrm{K}^{+}\right)$ and 396 chloride $\left(\mathrm{Cl}^{-}\right)$ions, giving a $1.12 \mathrm{M} \mathrm{KCl}$ concentration. Three replica simulations of 10 ns each were carried out at $0, \pm 0.2, \pm 0.5$, and $\pm 1 \mathrm{~V}$ mirroring a previous OmpF conductance study. ${ }^{(46)}$ We report the average and standard deviation of the current at each applied voltage by summing the movement of the charges in the $z$ direction. ${ }^{(46-48)}$

\section{Construction and Analysis of a G-Protein Coupled Receptor (GPCR) System}

The model of a GPCR was taken from the study by Hurst et al., ${ }^{(49)}$ in which the cannabinoid type 2 (CB2) receptor was simulated in a POPC bilayer. The receptor was extracted and rebuilt in a slightly larger simulation cell with 83/75 phospholipids and 53/53 2-arachidonoyl glycerol molecules (2-AG, an endogenous ligand for CB2) in the upper/lower leaflets. Three separate 100 ns simulations utilizing each of the three protocols were performed. Trajectories were saved at a $10 \mathrm{ps}$ time interval. Additional $1 \mathrm{~ns}$ simulations with trajectories saved every $100 \mathrm{fs}$ were also performed to provide a more accurate measure of the short-lived water hydrogen-bonding autocorrelation functions.

The hydrogen-bonding analysis and autocorrelation functions were computed using the LOOS ver 2.3.2 toolset. ${ }^{(50,51)}$ For each frame, a hydrogen bond is defined as present (1) or absent (0) 
using a given geometric criterion between pairs of donors and acceptors. The autocorrelation function is subsequently computed using one-half of the trajectory and averaged over all pairs. Distance/angle cutoffs of $2.5 \AA$ between the polar hydrogen and acceptor and a maximum deviation from linearity of $35^{\circ}$ were employed. In addition, an alternate approach to computing hydrogen-bond dynamics is given by a procedure introduced by Rapaport. ${ }^{(52)}$ Here, the hydrogen bond between an acceptor/donor pair, $h_{i j}$, is again defined as 1 or 0 on the basis of the above geometric criterion. The intermittent hydrogen-bond autocorrelation function is given by

$$
C_{I}(t)=\left\langle\frac{\sum h_{i j}\left(t_{0}\right) h_{i j}\left(t_{0}+t\right)}{\sum h_{i j}\left(t_{0}\right)^{2}}\right\rangle
$$

The sum in the above equation is over all hydrogen bonds that exist at time $t_{0}$. Intermittent refers to a definition where a given hydrogen-bond pair is allowed to break and subsequently reform. Finally, an averaging over multiple start times is performed. This approach produces an autocorrelation function that represents the hydrogen-bond population. In this work, the hydrogen-bond autocorrelation module of MDAnalysis ${ }^{(43)}$ was used to compute the above correlation function.

Construction and Analysis of L8 Peptide System

An ac- $\mathrm{L}_{8}$-nme (L8) peptide was constructed and embedded into the water phase of a box containing a preformed POPC lipid bilayer with an upper leaflet of 53 lipids and a lower leaflet of 52 lipids. The initial conformation was an ideal $\alpha$-helix, placed $10 \AA$ from the bilayer surface. The CHARMM36 protein force field was used to match previous simulations. ${ }^{(20,53)}$ Dihedral restraints were applied to the peptide backbone to maintain the helicity due to the high temperature used $(423 \mathrm{~K})$, which has been validated previously for the same system. ${ }^{(53,54)}$ The $200 \mathrm{kcal} /\left(\mathrm{mol} \mathrm{deg}^{2}\right)$ dihedral force constant used previously in a non-HMR system produced instabilities in the HMR system due to the smaller masses of the heavy backbone atoms, which are reduced by $\sim 15 \%$. Therefore, to maintain roughly the same mass-to-force-constant ratio, we similarly reduced the dihedral force constant to $175 \mathrm{kcal} /\left(\mathrm{mol} \mathrm{deg}^{2}\right)$ in the HMR system.

The insertion propensity, $p_{\mathrm{TM}}$, of the L8 peptide was calculated as the probability of the peptide being in the TM state. To distinguish the TM state from the S state, a criterion of $|z|<8 \AA$ was found to be optimal. The free energy of $\mathrm{S} \rightarrow \mathrm{TM}$ partitioning was then calculated as

$$
\Delta G_{\mathrm{S} \rightarrow \mathrm{TM}}=+k_{\mathrm{B}} T \log \left(1 / p_{\mathrm{TM}}-1\right)
$$

Construction of the Glycophorin A Dimer System

Simulations of glycophorin A (GpA) were started from the NMR structure in PDB 1AFO. The transmembrane (TM) helix dimer was placed in a POPC bilayer containing 60 lipids in each leaflet using CHARMM-GUI. ${ }^{(23)}$ The flexible termini of each protein were removed as done previously, ${ }^{(55)}$ leaving residues 69-97. The system was solvated with water, and $\mathrm{Na}^{+}$and $\mathrm{Cl}^{-}$ions were added at a concentration of $150 \mathrm{mM}$ (12 ions of each species).

MD Simulations 
After construction, HMR was applied to each unique system using a VMD ${ }^{(56)}$ script provided in the supplement, which created two copies, standard and modified, with the difference being the repartitioned mass in the latter. For the standard copy, MD was performed with a 2 fs time step, a $12 \AA$ cutoff (2-12) for the Lennard-Jones interactions, and a force-based switching function starting at $11 \AA$ to match that used for NAMD simulations in Klauda et al. (2010). ${ }^{(15)}$ The modified copy was simulated using a 4 fs time step and either a $12 \AA$ cutoff with switching starting at $11 \AA$ (4-12) or a $9 \AA$ cutoff with no switching (4-9). In all simulations, long-range electrostatic interactions were evaluated every 4 fs using the particle-mesh Ewald method. ${ }^{(57)}$ Unless otherwise stated, constant temperature was enforced using Langevin dynamics with a damping constant of $1.0 \mathrm{ps}^{-1}$, and constant pressure was enforced using a Langevin piston at $1 \mathrm{~atm} .{ }^{(58)}$ All simulations used NAMD2.12, ${ }^{(34)}$ TIP3P water, ${ }^{(59)}$ and, unless otherwise noted, the CHARMM36m force field for proteins ${ }^{(20,60)}$ and CHARMM36 for lipids. ${ }^{(15)}$ System visualization and analysis was performed with VMD. ${ }^{(56)}$ An example NAMD configuration file with the recommended settings for an HMR membrane simulation is provided in the supplement.

Free-Energy Calculations for the Glycophorin A Dimer System

Potentials of mean force (PMFs) for GpA separation were calculated for 2-12, 4-12, and 4-9 simulation protocols. When used, forced-based switching of the LJ interactions started at $10 \AA$ instead of $11 \AA$ used in other simulations. The PMFs are a function of the distance between the centers-of-mass of the $C_{\alpha}$ atoms of residues 72-96 of each helix. Replica-exchange umbrella sampling (REUS) was used. A total of 26 windows separated by $0.5-1 \AA$ were distributed along the range 7-24 $\AA$; the force constant in each window was between 2.5 and $4 \mathrm{kcal} / \mathrm{mol} \cdot \AA^{2}$. Typical exchange rates between windows were between 0.1 and 0.5 . For each of the three protocols, $100 \mathrm{~ns} /$ window was used $(2.6 \mu \mathrm{s} /$ protocol $)$; the first $10 \mathrm{~ns}$ was discarded with the weighted histogram analysis method (WHAM) performed on the last $90 \mathrm{~ns}{ }^{(61)}$ Although the PMFs are likely not yet fully converged, ${ }^{(55)}$ the root mean-square difference between 70 and 100 $\mathrm{ns}$ is $0.3 \mathrm{kcal} / \mathrm{mol}(2-12 ; 0.1$ for $90 \mathrm{vs} 100 \mathrm{~ns}), 0.9 \mathrm{kcal} / \mathrm{mol}(4-12 ; 0.2$ for $90 \mathrm{vs} 100 \mathrm{~ns})$, and 1.4 $\mathrm{kcal} / \mathrm{mol}(4-9 ; 0.5$ for $90 \mathrm{vs} 100 \mathrm{~ns})$.

\section{Results and Discussion}

To determine the effects of HMR, time step, and cutoff on structural and kinetic properties, we performed all-atom MD simulations employing three different simulation protocols. As a reference, we first simulated each system using a 2 fs time step and a 12 A cutoff with a forcebased switching function (referred to as 2-12 throughout the text). The other two protocols implement HMR along with a 4 fs time step and either a $12 \AA$ cutoff (referred to as 4-12) or a truncated $9 \AA$ cutoff with no switching (referred to as 4-9). Results of lipid membrane simulations are compared to observations from X-ray, neutron scattering, or NMR experiments by considering temporal and spatial averages of various observables, for example, bilayer thickness $\left(D_{\mathrm{HH}}\right)$ or APL. ${ }^{(62,63)}$

DPPC Membrane 
Dipalmitoylphosphatidylcholine (DPPC) is a common lipid that has been widely utilized in both experiments and simulation and thus has ample data for comparison. ${ }^{(15,64-66)} \mathrm{We}$ examined multiple static and kinetic properties of a DPPC membrane based on $100 \mathrm{~ns}$ simulations, including electron density, APL, $K_{\mathrm{A}}, S_{\mathrm{CD}}, D$, and dihedral trans-gauche transition rates.

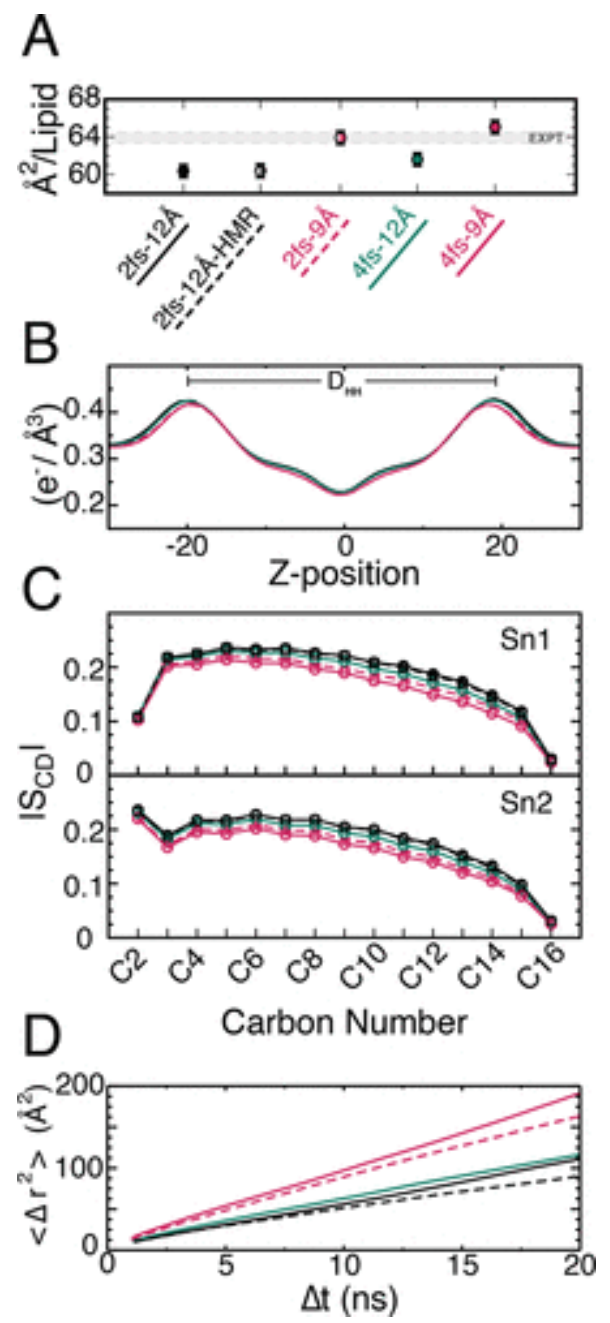

Figure 2. DPPC membrane properties. For each panel, results from 2-12 simulation is colored in black, 4-12 in green, and 4-9 in red. An additional 2-12 simulation with HMR is shown as a dashed black line, and an additional 2-9 simulation with a shorter cutoff is shown as a dashed red line. (A) Area per lipid with standard deviation bars taken from last $50 \mathrm{~ns}$ of production. (B) Plot of electron density; membrane thickness is measured from left peak to right peak of each distribution. (C) Plot of sn1 (top) and sn2 (bottom) lipid order parameters (circles). (D) Meansquared displacement versus time averaged over all lipids and times for each simulation.

First, static properties of the pure DPPC membrane were measured to compare with previous experimental and MD results. The APL for DPPC at $323 \mathrm{~K}$ in the 2-12 and 4-12 simulations is around $60-61 \AA^{2}$ (Table1). Experimental values are slightly larger at $63.3-64.3 \AA^{2}{ }^{(64,65)}$ Our numbers are in good agreement with those using HMR, a stochastic velocity rescaling thermostat and optimal temperature evaluation. ${ }^{(67)}$ However, Venable et al. found slightly larger simulated areas $\left(62.9-63.0 \AA^{2}\right)$ when using a switching function for the $L J$ interactions that began at $8 \AA$ 
instead of $10 \AA$, the latter being the default for the C36/C36m protein force field. ${ }^{(5)}$ In our 4-9 simulation, the APL increases substantially, to $65.1 \AA^{2}$. This increase is due to a reduction in the dispersion interactions, which are dominant in the aliphatic tails region (Figure S1), and is also reflected in the decreased tail order (Figure 2C) and thinning of the electron density profile (Figure 2B). Fluctuations in the APL contribute to the area expansion modulus, $K_{\mathrm{A}}$ (see eq 1). $K_{\mathrm{A}}$ values from each of the DPPC systems (Table1) are in reasonable agreement with the experimentally observed value $\left(231 \mathrm{mN} / \mathrm{m}^{(62)}\right)$, with no observable pattern between the different simulation protocols.

Table 1. Average Properties of Lipid Bilayer ${ }^{\mathrm{a}}$

\begin{tabular}{|c|c|c|c|c|c|}
\hline system & setting & $\operatorname{APL}\left(\AA^{2}\right)$ & $D_{\text {Hн }}(\AA)$ & $K_{\mathrm{A}}(\mathrm{mN} / \mathrm{m})$ & $D\left(\AA^{2} / \mathrm{ns}\right)$ \\
\hline \multirow[t]{6}{*}{ DPPC (323 K) } & $2 \mathrm{fs}-12 \AA$ & $60.4 \pm 0.7$ & 39.1 & 249 & 1.32 \\
\hline & 2 fs- $12 \AA$ HMR & $60.4 \pm 0.7$ & 39.0 & 228 & 1.04 \\
\hline & 2 fs $-9 \AA$ & $64.0 \pm 0.7$ & 38.0 & 196 & 1.96 \\
\hline & $4 \mathrm{fs}-12 \AA$ & $61.6 \pm 0.7$ & 38.7 & 253 & 1.37 \\
\hline & 4 fs- $9 \AA$ & $65.1 \pm 0.7$ & 38.0 & 251 & 2.27 \\
\hline & exp. & $63.1-64.2^{(64,65,68)}$ & $37.8-38.0^{(65,68)}$ & $231^{(62)}(318 \mathrm{~K})$ & $1.78(69)$ \\
\hline \multirow[t]{4}{*}{ POPE (310 K) } & $2 \mathrm{fs}-12 \AA$ & $57.4 \pm 0.6$ & 40.4 & 282 & 0.57 \\
\hline & 4 fs- $12 \AA$ & $58.0 \pm 0.7$ & 40.3 & 222 & 0.86 \\
\hline & 4 fs- $-9 \AA$ & $61.1 \pm 0.6$ & 39.5 & 280 & 1.11 \\
\hline & exp. & $59.8-60.8^{(70)}$ & $40.0^{(70)}(303 \mathrm{~K})$ & $233^{(71)}$ & \\
\hline \multirow[t]{4}{*}{ DOPC (295 K) } & $2 \mathrm{fs}-12 \AA$ & $67.0 \pm 0.6$ & 38.7 & 350 & 0.59 \\
\hline & 4 fs- $12 \AA$ & $67.0 \pm 0.6$ & 38.7 & 289 & 0.56 \\
\hline & 4 fs- $-9 \AA$ & $70.3 \pm 0.7$ & 38.0 & 241 & 1.04 \\
\hline & exp. & $67.4^{(68)}(303 \mathrm{~K})$ & $36.7^{(72)}(303 \mathrm{~K})$ & $300^{(73)}(303 \mathrm{~K})$ & $1.0^{(74)}(296.5 \mathrm{~K})$ \\
\hline \multirow[t]{4}{*}{ Top6 $(310 \mathrm{~K})$} & $2 \mathrm{fs}-12 \AA$ & $61.5 \pm 0.6$ & 37.2 & 327 & 0.71 \\
\hline & 4 fs- $12 \AA$ & $62.5 \pm 0.6$ & 37.0 & 269 & 0.84 \\
\hline & 4 fs- $9 \AA$ & $64.8 \pm 0.6$ & 36.9 & 290 & 1.35 \\
\hline & exp. & $63.0 \pm 0.2^{(75)}$ & & $240^{(75)}(310 \mathrm{~K})$ & \\
\hline
\end{tabular}

${ }^{a}$ Area per lipid (APL), membrane thickness $\left(D_{\mathrm{HH}}\right)$, area compressibility modulus $\left(K_{\mathrm{A}}\right)$, and diffusion coefficient $(D)$. The values of temperature in the parentheses indicate that the property was measured at that specific temperature. We note that for all simulations presented in this table, temperature was controlled using Langevin dynamics with a damping constant of $1.0 \mathrm{ps}^{-1}$.

Next, we looked at order parameters (Figure 2C), finding that the values from our 2-12 simulation agree well with previous experimental ${ }^{(64)}$ and simulation ${ }^{(15,76-78)}$ results. When comparing the 4-12 simulation to the 2-12 simulation, there is a small decrease in lipid order resulting in a $5.7 \pm 2.8 \%$ difference on average over all of the carbon positions; when comparing 2-12 and 4-9 simulations, the decrease is much larger $(17.4 \pm 6.4 \%)$, suggesting that the cutoff has a significant effect on tail order. To decipher the individual roles of HMR and the $4 \mathrm{fs}$ time step, we performed an additional simulation with 2 fs time step, 12 Å cutoff, and HMR (2-12HMR), which showed a decrease of $1.0 \pm 0.8 \%$ on average in the order parameters (Figure $2 \mathrm{C}$ ), similar to the 2-12 simulation. This result suggests that HMR has little to no effect on order parameters, a 4 fs time step causes a slight but measurable decrease, and a reduced cutoff causes a substantial decrease in lipid tail order. The decrease in tail order parameters is also evident from an increased interdigitation of the aliphatic tails in the 4-9 simulation (Figure S1, bottom) as compared to the 2-12 (Figure S1, top) and 4-12 (Figure S1, middle) simulations. Furthermore, 
aliphatic interdigitation between membrane leaflets results in membrane thinning by $1 \AA$ in the 4-9 simulation as compared to the 2-12 simulation (Figure $2 \mathrm{~B}$ ).

\section{Diffusion in the DPPC Membrane}

We examined the rate of lipid diffusion as a function of HMR, time step, and cutoff. The diffusion constant is known to be sensitive to a number of simulation parameters, including box size $^{(79)}$ and thermostat. ${ }^{(80)}$ A high sensitivity was observed here as well. Diffusion constants were calculated from the slope of the mean-square displacement versus time, averaged over lipids and time (Figure 2D). We found that the 2-12 and 4-12 simulations produced similar values at 1.32 and $1.37 \AA^{2} / \mathrm{ns}$, respectively, both smaller than the experimental value of $1.78 \AA^{2} / \mathrm{ns} .{ }^{(69,81)}$ For the 4-9 simulations, $D$ was much larger at $2.27 \AA^{2} /$ ns. All values of $D$ described in this section can also be found in Table S2.

To further disentangle the roles of cutoff, time step, and HMR, we carried three additional simulations: one with a 2 fs time step but with HMR applied (2-12-HMR), one with a 2 fs time step and a $9 \AA$ cutoff (2-9), and one with a 2 fs time step and $12 \AA$ cutoff as well as an area fixed to match that of 4-9 simulation (2-12-CA; 65.1 $\AA^{2} /$ lipid). The $2-12-H M R$ simulation produced a value of $D$ at $1.04 \AA^{2} / \mathrm{ns}$, even smaller than 2-12 (1.32 $\left.\AA^{2} / \mathrm{ns}\right)$, indicating that mass repartitioning is not a source of increased diffusion observed in the 4-9 simulation. The 2-9 simulation gave a value of $D$ at $1.96 \AA^{2} / \mathrm{ns}$, close to 4-9 and much greater than 2-12 and 4-12. This suggests that the decreased cutoff, which results in an increase in APL, is responsible for a large fraction of the increase in $D$. Finally, the 2-12-CA simulation resulted in a value of $D$ of $1.54 \AA^{2} / \mathrm{ns}$, closer to 212 than to 4-9, suggesting that the increase in $D$ observed in the 4-9 simulation is primarily a direct result of the reduced cutoff, as opposed to an indirect result due to the increased APL.

The previously described simulations all used Langevin dynamics with a damping constant of $\gamma$ $=1.0 \mathrm{ps}^{-1}$. However, although it is one of the most common thermostats used in NAMD simulations, Langevin dynamics has been demonstrated to reduce diffusion constants by up to $35 \% .{ }^{(80)}$ We decided to investigate the role of the thermostat by first lowering the Langevin damping constant from 1.0 to $0.1 \mathrm{ps}^{-1}$. Unsurprisingly, $D$ became larger for all systems at 1.55 $\AA^{2} / \mathrm{ns}$ for $2-12,2.30 \AA^{2} / \mathrm{ns}$ for $4-12$, and $3.59 \AA^{2} / \mathrm{ns}$ for $4-9$. We also tested the Lowe-Andersen thermostat, which is designed to minimize suppression of diffusion. ${ }^{\left({ }^{82}\right)}$ Interestingly, the 2-12 simulation gave a similar value of $D$ at $1.23 \AA^{2} /$ ns (Figure S2). However, $D$ was much greater for 4-12 at $2.33 \AA^{2} / \mathrm{ns}$ and $4-9$ at $3.89 \AA^{2} / \mathrm{ns}$, similar to Langevin dynamics with a very small damping constant. The increase in $D$ when going from a 2 to a 4 fs time step, which was minimal previously (see Table1), is significant here. Increasing the time step is expected to contribute a very small additional "numerical damping" when using Langevin dynamics, ${ }^{(83)}$ counter to what is observed. Alternatively, it has been found previously in simulations of a generic van der Waals fluid that increasing the time step leads to enhanced diffusion, due to harder collisions. ${ }^{(84)}$ This is also borne out by looking at the average temperatures, which are consistently higher for a 4 fs time step versus a 2 fs one (see Figure S3 and Table S3).

To investigate another possible source for the different diffusion constants related to the altered masses, the trans-gauche $(\mathrm{t}-\mathrm{g})$ transition rate for the aliphatic dihedral angles was measured. It has been suggested that intramolecular conformation of the lipid molecule is closely related to 
the intermolecular structure of the membrane, such that the $\mathrm{t}-\mathrm{g}$ transition time in dihedral angles may provide a good estimate for the equilibration time needed. ${ }^{(85)}$ Here, we measured the rate of transition between the trans, gauche + , and gauche- conformations of sequential carbons on the lipid tails of DPPC. It was found that the transition rates between trans, gauche+, and gauchewere nearly identical for all simulation protocols over a $1 \mathrm{~ns}$ simulation (see Tables S4 and S5). Therefore, the variation in diffusion constants among the three simulation protocols described above did not correlate with their trans-gauche transition rates.

\section{Other Membranes}

After measuring the effects of HMR, longer time step, and a shorter cutoff on an unsaturated membrane, we proceeded to simulate three additional membranes to determine if varying lipid composition would alter our initial observations. We performed simulations of 1-palmitoyl,2oleoyl-sn-glycero-phosphatidylethanolamine (POPE, one unsaturated tail), 1,2-oleoyl-snglycero-phosphotidylcholine (DOPC, two unsaturated tails), as well as the so-called "Top6" membrane, which is a mixture of saturated, unsaturated, and cyclic-containing lipids. ${ }^{(40)}$ To expand upon the same measurements as the DPPC simulations, each of these membranes was simulated using the 2-12 protocol as a control, as well as the additional 4-12 and 4-9 protocols applying HMR. We report the various physical properties of the membranes in Table1. These properties were calculated over the last $50 \mathrm{~ns}$ of each $100 \mathrm{~ns}$ simulation.

For each system, APL values in the 2-12 and 4-12 systems are in a good agreement with experimental measurements (see Figure S4 for graphs of APL fluctuation over the entire simulation period). It was also observed that the 4-9 systems have the largest APL values in each of the systems, demonstrating again that APL increases with a shorter cutoff (12 vs $9 \AA$ ). One particular result of interest is the difference in diffusion coefficients observed in each of the membrane-only systems. For the POPE membrane, we observe a 50\% increase going from 2-12 to 4-12, although both DOPC and Top6 membranes are closer (5-18\% difference between 2-12 and 4-12); however, as was observed for the DPPC membrane, damping from the Langevin thermostat may be suppressing larger differences between them. All 4-9 systems have diffusion values nearly twice as large as their respective 2-12 system. This is due in part to the increased fluidity of the membrane as is also seen in the lipid order parameters (Figures S5-S8). Unlike other properties, the values of $K_{\mathrm{A}}$ showed no consistent pattern between simulation protocols, although almost all numbers were within a range of $\sim 200-300 \mathrm{mN} / \mathrm{m}$.

\section{Lipid Mixing and Membrane Bending Modulus}

To examine the impact of time step, HMR, and cutoff on lipid mixing, we turn to the POPC:CHL mixture previously investigated by microsecond Anton simulations. ${ }^{(42)}$ Each leaflet of the mixture was composed of 70 POPC and 35 cholesterol, with the latter initially placed at the center of the bilayer. This mixed membrane was simulated for $1 \mu \mathrm{s}$ with either the 4-9 or the 412 protocol (the Anton simulation reported previously ${ }^{(42)}$ provides the reference for 2-12). Unless otherwise noted, we analyzed the trajectory from the first microsecond of the $2 \mu$ s Anton simulation for a fair comparison with the $1 \mu$ s runs performed in this work. 
As shown in Figure 3, regardless of the protocol used, the final structures of the mixtures are similar to each other, as reflected by the ratios of unlike-to-like (UL) neighbors (Figure 3A,B) and the size distribution of cholesterol clusters (Figure 3C). Therefore, the equilibrium distribution of the lipids is unaffected by the choice of time step, use of HMR, or cutoff. The APL of the mixtures, however, is clearly affected: averaging over all POPC and cholesterol yields an APL of 46.4, 47.1, and 49.4 $\AA^{2}$ for the 2-12, 4-12, and 4-9 simulations, respectively, reflecting a trend consistent with that observed in our DPPC simulations (Table1). Comparison of the three simulations also reveals a clear difference in the speed of mixing.

Semiquantitatively, this can be seen from Figure 3D-F and Figure S9, which show the evolution of the radial pair distribution function $g(r)$ over the course of the 4-9 and the 4-12 simulations as well as the first $1 \mu$ s of the previously performed 2-12 simulation. The $g(r)$ curves, drawn every $100 \mathrm{~ns}$, suggest that the 4-9 run converges much faster than the other two simulations. Quantitatively, the lateral diffusion coefficient of cholesterol in the 4-9 simulation $\left(0.65 \AA^{2} / \mathrm{ns}\right)$ is over $2 \times$ larger than that in the $2-12$ simulation $\left(0.25 \AA^{2} / \mathrm{ns}\right)$. Similarly, POPC diffuses significantly faster in the former system, with a diffusion coefficient of $0.61 \AA^{2} / \mathrm{ns}\left(0.22 \AA^{2} / \mathrm{ns}\right.$ in the 2-12 run). In the 4-12 simulation, diffusion of cholesterol $\left(0.44 \AA^{2} / \mathrm{ns}\right)$ and POPC $(0.30$ $\left.\AA^{2} / \mathrm{ns}\right)$ is also accelerated as compared to the 2-12 simulation, although to a much smaller degree than in the 4-9 simulation.
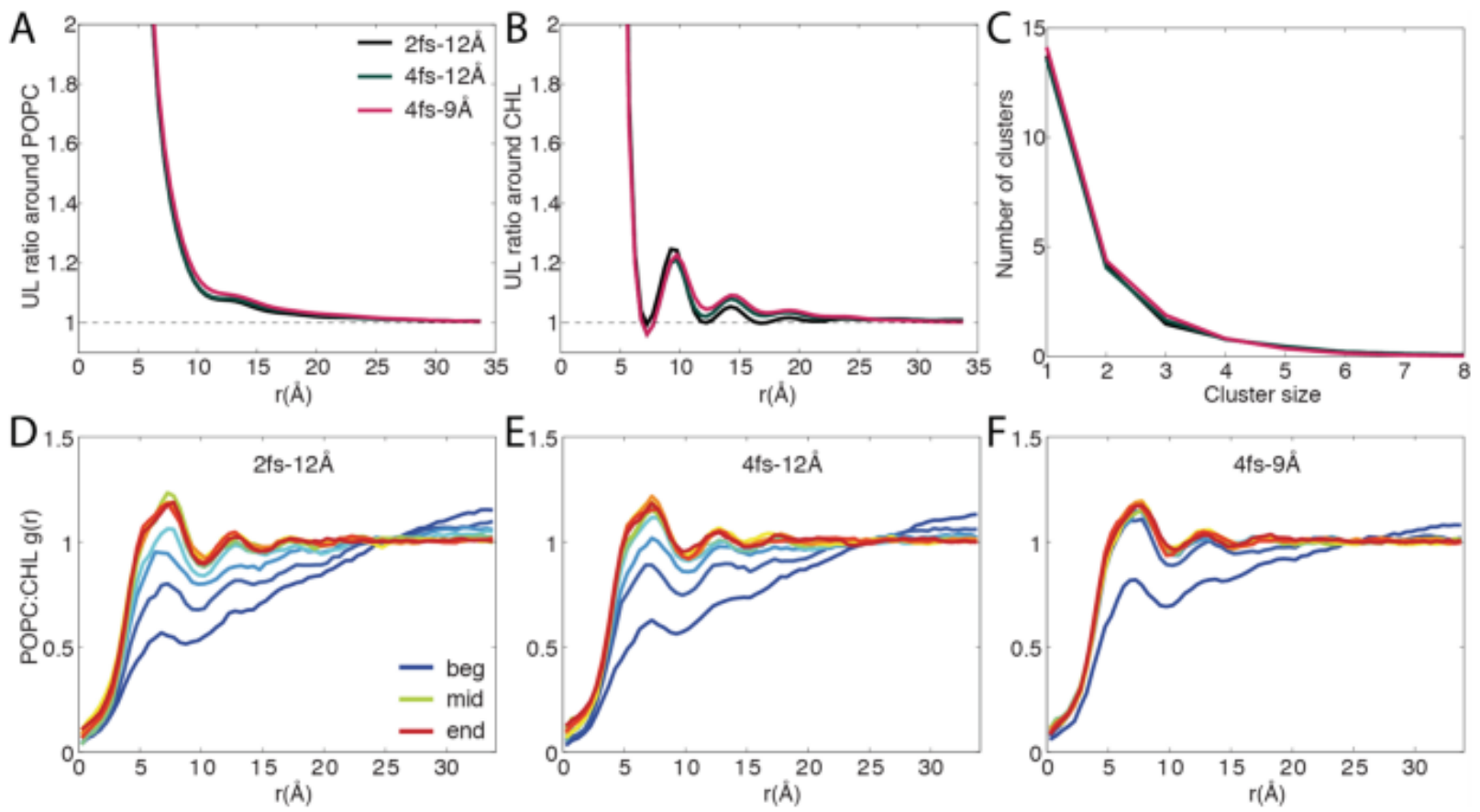

Figure 3. Impact of HMR, time step, and cutoff on lipid mixing. (A,B) Average ratio of unlike neighbors to like neighbors (UL) around a given lipid species. The expected values based on mixing ratio are indicated by dashed lines. (C) Clustering analysis results of the POPC:CHL mixture. (D-F) Time evolution of the radial pair distribution functions $g(r)$ for POPC:CHL. $g(r)$ is averaged in $100 \mathrm{~ns}$ blocks and colored by simulation time, with blue, green, and red indicating the beginning, the middle, and the end of a simulation, respectively.

Apart from lipid lateral diffusion, it is worth noting that a cholesterol flip-flop event was recorded in both the 4-9 and the 4-12 simulations, in contrast to zero flip-flop events recorded 
throughout the $2 \mu$ s 2-12 simulation. The larger APL in the 4-12 and 4-9 simulations may have contributed to their increased cholesterol flip-flop events, as the APL is linked to the free-energy barrier of defect formation in a membrane. ${ }^{\left({ }^{86}\right)}$ Taken together, the choice of time step, cutoff, and use of HMR has a negligible effect on the distribution of lipids in an equilibrated mixture, although other equilibrium properties, such as the APL, are evidently affected. On the speed of lipid mixing, while increasing the simulation time step from 2 to 4 fs and applying HMR has a rather moderate effect, decreasing the cutoff from 12 to $9 \AA$ significantly accelerates mixing. Overall, our results indicate that mixing simulations designed to investigate the equilibrium distribution of various lipid components can safely employ HMR.

Following our investigation on lipid mixing, we went on to evaluate how HMR may affect one of the most important material moduli of a membrane, its bending modulus. Using a bilayer with 680 POPC lipids, we performed three $1 \mu$ s simulations with the 2-12, 4-12, and 4-9 protocol, respectively. The $\sim 150 \AA \times 150 \AA$ bilayer supported relatively long-wavelength undulation modes, thereby allowing us to determine $k_{\mathrm{c}}$ from $\left\langle u^{2}(q)\right\rangle$, the average square amplitude of undulation at a given wavenumber $q$. More specifically, $k_{\mathrm{c}}$ was calculated from the last $900 \mathrm{~ns}$ of the $1 \mu$ s trajectories according to $\left\langle u^{2}(q)\right\rangle=k_{\mathrm{c}} k_{\mathrm{B}} T A^{-1} q^{-4}$ using the MDAnalysis package ${ }^{(43)}$ and methods presented in refs (87) and (88). As shown in Figure S10, $k_{\mathrm{c}}$ was found to be $30.9 k_{\mathrm{B}}$ $\left(12.9 \times 10^{-20} \mathrm{~J}\right)$ in the 2-12 simulation, which is comparable to the experimental value $\left(9.0 \times 10^{-}\right.$ ${ }^{20} \mathrm{~J}$ ) reported for a similar lipid bilayer (1-stearoyl-2-oleoyl-sn-glycero-3-phosphocholine). ${ }^{(89)}$

To estimate the uncertainty in $k_{\mathrm{c}}$, we first examined the blocked standard error in $\left\langle u^{2}(q)\right\rangle$. The undulation mode with the smallest wavenumber (longest wavelength) was found to have the largest error, calculated from the last $900 \mathrm{~ns}$ (Figure S10). The error is approximately an order of magnitude greater if only the last $50 \mathrm{~ns}$ of trajectories are used in the analysis instead of the last $900 \mathrm{~ns}$ (data not shown). This behavior supports the need for microsecond-long trajectories in reliable analysis of $k_{\mathrm{c}}$. Here, the uncertainty in our $k_{\mathrm{c}}$ values was found to be approximately $2 k_{\mathrm{B}} T$. As compared to the 2-12 run, $k_{\mathrm{c}}$ decreased slightly to $28.6 k_{\mathrm{B}} T$ in the 4-12 simulation. In the 4-9 simulation, a further decrease was seen, with $k_{\mathrm{c}}$ reaching $25.4 k_{\mathrm{B}} T$. We note that the difference between the 2-12 and the 4-9 simulations is well beyond the estimated uncertainty in $k_{\mathrm{c}}$, indicating that the comparison is statistically meaningful. The average projected APL was found to be $64.2,64.9$, and $67.3 \AA^{2}$ in the $2-12,4-12$, and $4-9$ simulations, respectively. Taken together, these results again reflect the weakened lipid interactions when a short cutoff (9 $\AA$ ) is adopted. Such weakened interactions not only produce an increased area per lipid, but also reduce the energetic cost of bending the membrane, thereby resulting in a decreased $k_{\mathrm{c}}$.

\section{Electric Field Simulations of OmpF}

One important function of membrane proteins is to regulate the flow of ions into and out of the cell. OmpF is a well-characterized trimeric protein that acts as a nonspecific ion channel in the outer membrane of Gram-negative bacteria. ${ }^{(90,91)}$ To continue the investigation into the effect of time step, cutoff, and HMR on properties like conductance, we performed simulations with an applied electric field on the outer membrane ion channel OmpF. We note here again that HMR was never applied to water molecules. Scaling by molarity, our results across all simulation protocols compare favorably to those reported by Pezeshki et al., ${ }^{(46)}$ including the higher current for positive voltages due to a slight cation selectivity of the channel. At low voltages, we observe 
a near exact agreement between all three simulation protocols (Figure 4). At higher voltages, the 4-9 simulation underestimates the current, while the 4-12 simulation slightly overestimates the current with respect to the conventional 2-12 simulation. However, the deviation for each of the HMR systems is within, or very nearly within, the standard deviation of the conventional 2-12 system, indicating that HMR, longer time step, and shorter cutoff do not significantly affect the conductance of OmpF. Interestingly, however, the root mean-square deviation (RMSD) of the proteins is notably higher in most cases for the 4-9 simulations, especially at higher voltages, while the 2-12 and 4-12 simulations are practically the same (Figure S11). This suggests that the shorter cutoff may perturb the structure of proteins under applied forces.

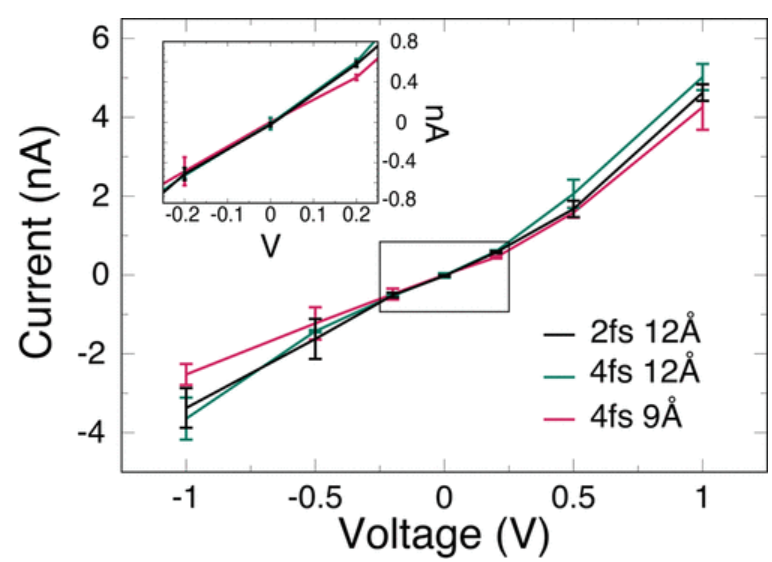

Figure 4. Current versus voltage for OmpF membrane protein systems (2-12 in black, 4-12 in green, and 4-9 in red). Inset graph shows the low-potential regime.

\section{CB2 Simulations}

GPCRs are integral membrane proteins that share a common architecture of seven transmembrane helices (TMHs) connected by intracellular (IC) and extracellular (EC) loops. These membrane-bound proteins are central among the classes of proteins involved in signal transduction. Ligand binding to, and subsequent conformational changes of, GPCRs leads to activation of intracellular heterotrimeric G-proteins and ultimately cellular response. To a large degree, this functionality is achieved by the inherent flexibility of GPCRs. ${ }^{(92)}$ As a result, functional outcomes can and are modulated by their lipid environment. ${ }^{(93)}$ Therefore one should anticipate that the structure and function of these membrane bound systems would be coupled to the details of the treatment of the membrane environment. Given the importance of hydrogen bonding in maintaining the structure of GPCRs in a membrane environment, we have explored the effects of HMR, as well as potential energy truncation, on the initial equilibration of the receptor to a lipid environment, as well as the occurrence and lifetime of intra- and intermolecular hydrogen bonding for the CB2/POPC systems.

Using the CB2 model of Hurst et al., ${ }^{(49)}$ we have run three 100 ns simulations for each of the 212, 4-12, and 4-9 protocols. The RMSD of the transmembrane portion of the receptors is reported in Figure 5. The use of the transmembrane region for analysis is motivated by the work of Grossfield et al., ${ }^{(94)}$ where they have shown that sampling on the time scale of $100 \mathrm{~ns}$ for the extracellular and intracellular loops of rhodopsin has not converged. In fact, further analysis indicates that convergence can be expected to be much longer. ${ }^{(95)}$ Here, we are interested 
primarily in the initial equilibration of the starting structure. For each trajectory, the first $20 \mathrm{~ns}$ was discarded, and all further analysis was performed on the last $80 \mathrm{~ns}$. Subsequently, time averaging for each trajectory was performed. This resulted in an average RMSD of $1.41( \pm 0.22)$ $\AA, 1.40( \pm 0.23) \AA$, and $1.53( \pm 0.14) \AA$ for the $2-12,4-12$, and 4-9 simulation protocols, respectively (standard deviation in parentheses). From these data, it appears the choice of HMR and time step produces results that are nearly identical on the $100 \mathrm{~ns}$ time scale. The 4-9 simulation has a slightly larger RMSD; however, given the standard deviations, these differences appear minimal.

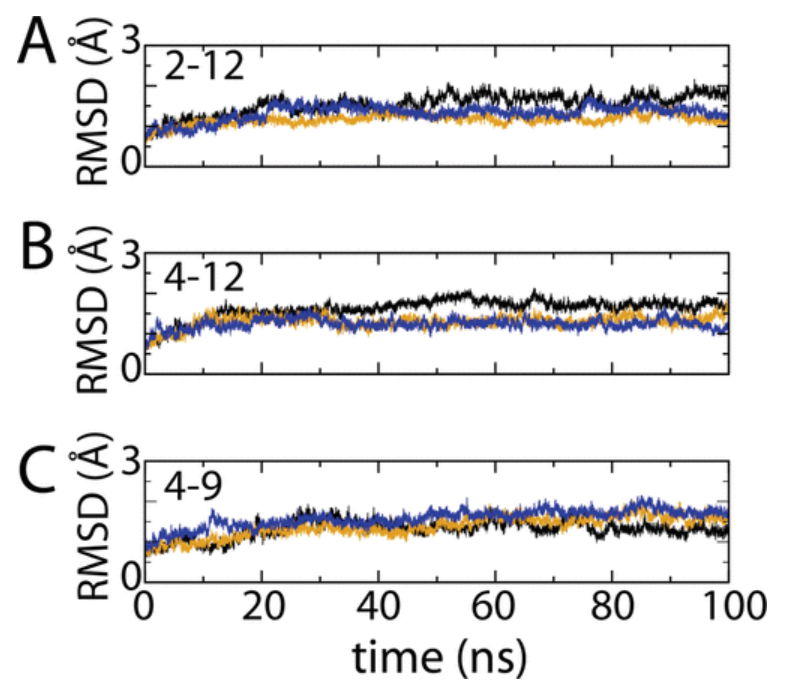

Figure 5. Root mean-square deviation (RMSD) for the three 100 ns trajectories using (A) the 212, (B) the 4-12, and (C) the 4-9 protocols. The colors for the traces represent independent runs.

We examined the hydrogen-bonding capacity of the transmembrane helices as a function of HMR, time step, and cutoff. In Figure S12, the fraction of hydrogen bonds is plotted for each transmembrane helix in each protocol. Overall, given the overlap of the error bars, the three simulation protocols produce essentially the same helical hydrogen-bonding patterns. Last, the functionally important "ionic lock", a salt bridge between the intracellular ends of TMH3-TMH6 keeping the receptor in the inactive state, is maintained, and the toggle switch residue W6.48 remains in the $\mathrm{g}+$ conformation for all three sets of simulations. ${ }^{(92,96)}$

We also examined the intra- and intermolecular hydrogen-bonding autocorrelation functions. Given that water was not subjected to HMR and polar hydrogens are not present in POPC, hydrogen bonding between CB2 donors (polar hydrogens) and the available acceptors in the simulation, including CB2, water, and lipid acceptors, has been considered. Because of the short lifetime of a typical water hydrogen bond, we ran and analyzed separate $1 \mathrm{~ns}$ simulations with a high trajectory output rate. The averaged autocorrelation functions obtained using LOOS are reported in Figure 6A-C. These individual correlation functions appear to give similar results, with all three protocols agreeing to within their standard deviations.

In addition to the LOOS-based hydrogen-bond autocorrelation functions, MDAnalysis ${ }^{(43)}$ was used to generate population autocorrelation functions, ${ }^{(52)}$ which are plotted in Figure 6D-F. Analogous to the LOOS results, and given the standard deviations, there is very little apparent 
difference in the autocorrelation functions indicating that the hydrogen-bonding dynamics for these $100 \mathrm{~ns}$ trajectories are not particularly sensitive to the mass repartitioning/cutoff treatment.
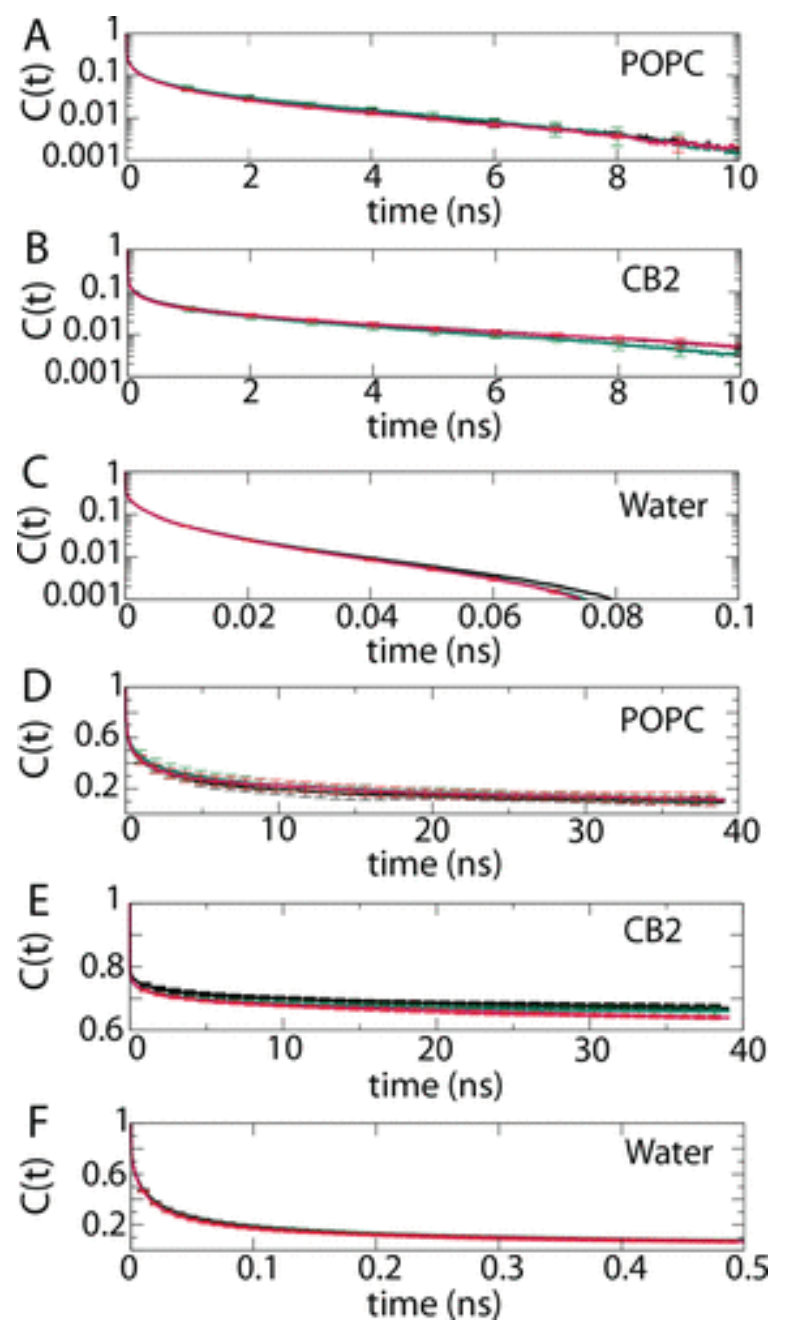

Figure 6. Hydrogen-bonding autocorrelation functions. 2-12 data are shown in black, 4-12 in green, and 4-9 in red. Values reported are averaged over three separate runs with the standard deviations reported. (A-C) Hydrogen-bonding correlation functions generated with LOOS for the indicated interactions, plotted using a logarithmic scale on the $y$-axis. (D-F) Hydrogenbonding correlation functions generated with MDAnalysis.

Peptide Partitioning in POPC

To further investigate how membrane-protein kinetics are affected by HMR, we performed long equilibrium simulations of an octoleucine (L8) helix embedded within a POPC bilayer (see Methods) using the 4-12 and 4-9 protocol for comparison to a previous simulation on Anton. ${ }^{(53)}$ With the peptide starting in a transmembrane (TM) state and using a high temperature $(423 \mathrm{~K})$, we measured the number of transitions between the TM and surface-associated (S) states over the course of a 1.3 and $1.5 \mu$ s production run for the 4-12 and 4-9 protocol, respectively. The TM state was defined as $|z|<8 \AA$, where $z$ is the distance between the center of mass of the peptide backbone and the center of the membrane, consistent with previous studies 
of L8 insertion into a lipid bilayer. ${ }^{(53)}$ The high temperature was necessary for sufficient sampling of the two states, and backbone dihedral restraints were added to ensure the peptide did not unfold (see Methods). ${ }^{(53)}$ We observed 14 transitions in $1.3 \mu \mathrm{s}(10.7$ transitions/ $\mu \mathrm{s})$ in the 412 simulation, while we only observed 10 transitions in $1.5 \mu \mathrm{s}(6.7$ transitions/ $\mu \mathrm{s})$ in the 4-9 simulation (see Figure 7A). In addition, the peptide spent far less time in the S state for the latter, with $\Delta G_{\mathrm{S} \rightarrow \mathrm{TM}}=-2.8 \mathrm{kcal} / \mathrm{mol}$ for the $4-9$ simulation as compared to $-0.9 \mathrm{kcal} / \mathrm{mol}$ for the $4-12$ simulation (see Figure $7 \mathrm{~B}$ and Methods for calculation of $\Delta G_{\mathrm{S} \rightarrow \mathrm{TM}}$ ). Previous multi-

microsecond-scale simulations of L8 at $423 \mathrm{~K}$ run on the Anton supercomputer with a $2.5 \mathrm{fs}$ time step and a 13-14 $\AA$ cutoff without HMR produced roughly 12 transitions/ $\mu$ s, with a temperatureindependent $\Delta G_{\mathrm{S} \rightarrow \mathrm{TM}}=-0.9 \pm 0.2 \mathrm{kcal} / \mathrm{mol}^{(53)}$ Results from the 4-12 simulation are in good agreement with these values, reproducing the free-energy difference and only slightly underestimating the $\mathrm{S} \rightarrow$ TM transition rate. The 4-9 simulation, however, significantly overestimates the free-energy difference and underestimates the transition rate. This difference is likely due to the increase in area (6\% on average for the 4-9 vs the 4-12 simulation), which may stabilize the TM state.
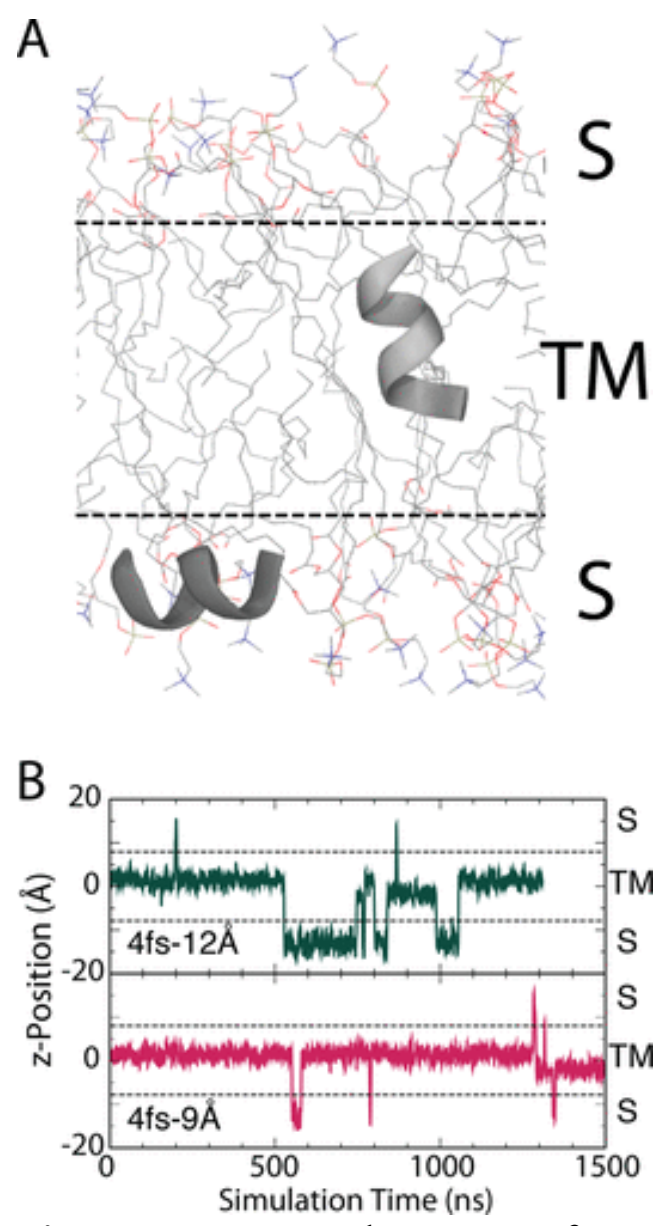

Figure 7. Transmembrane to surface-associated transitions of L8 helix in a POPC bilayer with HMR. (A) Position and orientations of L8 in surface-associated (S) and transmembrane (TM) states. The peptide is shown as a cartoon representation and colored gray. Lipid molecules are shown in line representation and colored by atom name (hydrogen atoms omitted). (B) Position of helix within the membrane for the (top) 4-12 simulation and (bottom) 4-9 simulation. The center of the membrane is defined as $z=0 \AA$, and TM states are defined as $|z|<8 \AA$. 
Free-Energy Calculations of Transmembrane Helix Dimerization

The transmembrane domain of glycophorin A $(\mathrm{GpA})$, a single helix, forms a dimer in the membrane through the interactions of matched GxxxG sequences in each copy. It has long been a model system for both experimental and computational investigations into helix-helix packing in the membrane. ${ }^{(55,97-107)}$ A number of these studies focused on the energetics of dimerization, calculating potentials of mean force (PMFs) in multiple environments and using different reaction coordinates. The most straightforward choice is a center-of-mass distance between the two helices, although it has been recognized recently that this coordinate becomes degenerate at close $(<15 \AA)$ separation. ${ }^{(107)}$ Nonetheless, for simplicity of comparison to previous PMFs, we used the center-of-mass distance between the two helices to calculate the PMF here for each of the 2-12, 4-12, and 4-9 protocols.

PMFs were determined using replica-exchange MD with 26 windows covering a range of 7-24 $\AA$ (see Methods). All PMFs exhibit a minimum at $7.5 \AA$ with a well depth of $\sim 6 \mathrm{kcal} / \mathrm{mol}$, similar to at least one of the previously determined PMFs (Figure 8). ${ }^{103)}$ While the 2-12 and 4-12 PMFs are broadly similar (root mean-square difference of $0.5 \mathrm{kcal} / \mathrm{mol}$ ), the 4-9 PMF stands out, reaching its maximum roughly 5-10 $\AA$ closer than the other two (root mean-square difference between 4-9 and 2-12 PMFs of $1.0 \mathrm{kcal} / \mathrm{mol}$ ). Thus, we see here that the shorter cutoff has a more dramatic effect on the free energy of interaction than the longer time step and HMR.

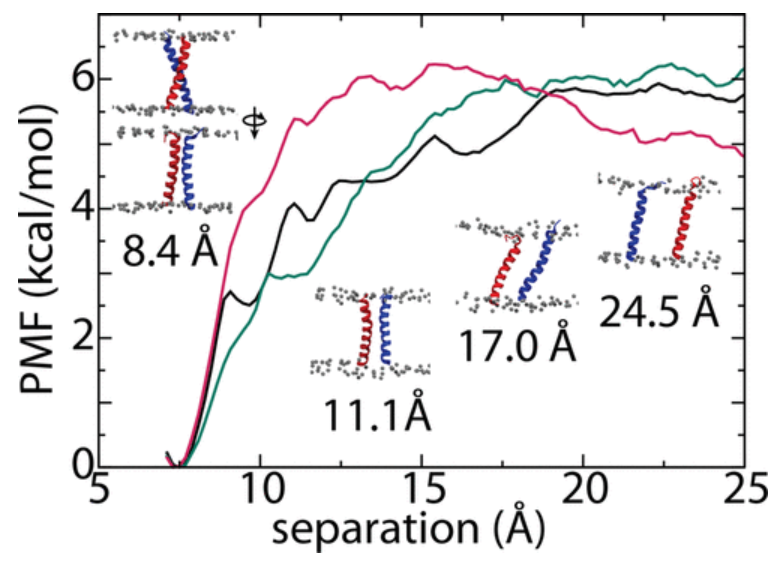

Figure 8. Potentials of mean force for glycophorin A separation as a function of distance between the centers-of-mass of the transmembrane helices (100 ns/window REMD calculation). Black is the 2-12, green is the 4-12, and red is the 4-9 simulation.

Benchmarks

Benchmarking simulations were carried out for the 240-lipid DPPC membrane (113 064 atoms) and the large, 680-lipid POPC membrane (170 844 atoms). Each system was run on 1, 2, 4, 8, 12, 16, and, for the larger system, 24 CPU-only nodes of (1) Stampede2 at the Texas Advanced Computing Center (Intel Xeon Skylake CPUs; 48 cores/node) and (2) Bridges at the Pittsburgh Supercomputing Center (Intel Haswell CPUs; 28 cores/node). Each simulation system was run using (1) the 2-12 protocol, (2) the 4-12 protocol, (3) the 4-12 protocol and PME evaluated every 8 fs, (4) the 4-9 protocol, and (5) the 4-9 protocol and PME evaluated every 8 fs. 
As expected, the 4-9/8 fs-PME simulations had the greatest simulation output (ns/day) at practically all node counts (Figure S13). However, the benefits accruing from each approximation were not equal. The $4-12$ simulations are consistently $\sim 75 \%$ faster than the $2-12$ simulations on CPUs (Figure S14). However, employing a $9 \AA$ cutoff only speeds up simulations by an additional 20-45\%. Evaluating PME electrostatics every 8 fs instead of $4 \mathrm{fs}$ is of mixed benefit at either cutoff, giving at most $40 \%$ improvement in speed at high node counts; at reasonable node counts (efficiency $>75 \%$ ), the speedup is $20 \%$ at most (Figure S15).

Although no production simulations in this Article used GPUs, we also benchmarked on 1-4 NVIDIA GTX 980 cards on a single node with two Intel Xeon Haswell CPUs (24 cores, using 6 cores/GPU). Going from 2 to 4 fs time steps gives a consistent speedup of $40 \%$. No improvement was seen when shortening the cutoff; short-range interactions are evaluated on the GPU(s), but these simulations are CPU-limited. Additionally, no benefit was seen when evaluating PME every 8 fs instead of 4 fs (Figure S13E,F). For comparison, we tested the DPPC membrane with Amber 16 on a single P100 GPU. As with NAMD on CPUs, going from a 2 to a 4 fs time step gave a speedup of $85-90 \%$. In contrast, however, the $9 \AA$ cutoff with no switching gave an additional $60 \%$ speedup.

\section{Conclusions}

MD simulations of membranes and membrane proteins have become increasingly common over the last two decades, and the need for longer trajectories has grown concomitantly. Thus, methods to improve the efficiency of these simulations are highly desirable. One approach, although at least two decades old, ${ }^{(26)}$ has gained prominence recently: HMR. HMR accelerates the simulation by redistributing the mass from a parent atom onto its bonded hydrogens, thus slowing their motions and permitting a larger time step, typically 4 fs instead of $1-2 \mathrm{fs} .{ }^{(27)} \mathrm{We}$ applied HMR to a variety of membrane-only and membrane-protein systems using NAMD ${ }^{(34)}$ along with the $\mathrm{C} 36 / \mathrm{C} 36 \mathrm{~m}^{(15,20,60)}$ force field. Our results show only marginal differences between the standard masses with a 2 fs time step and the repartitioned masses with a 4 fs time step for almost all properties tested, the electron density profiles, order parameters, and compressibility modulus. APL and diffusion constants, which showed some differences, are discussed below. The overall robustness of MD simulations to such system alterations is supported by the modified virtual interaction sites method in Gromacs, which also found excellent agreement for most structural properties of modified C36 lipids using a time step as large as 5 fs. ${ }^{(33)}$

Diffusion constants were found to be larger in many cases with a 4 fs time step as compared to a 2 fs one. Specifically, $D$ was as much as $50-90 \%$ greater in the 4-12 DPPC simulation as compared to the 2-12 simulation when Langevin dynamics with a damping constant of $0.1 \mathrm{ps}^{-1}$ or a Lowe-Andersen thermostat is used (see Figure S2); however, this difference was nonexistent in most cases with Langevin dynamics with a damping constant of $1.0 \mathrm{ps}^{-1}$. Such an increase is expected due to harder collisions that occur when atoms are allowed to move closer to one another in a single time step. ${ }^{(84)}$ The effect of harder collisions is also manifest in the temperature (see Figure S3 and Table S2) and the APL, which was slightly larger (by 1-2\%) in most systems with a 4 fs time step, similar to the increase in area observed when going from 1 to 2 fs time steps. ${ }^{(5)}$ While to obtain precise kinetic properties, it is recommended to first test the 
simulation parameters selected against experimental data, a general approach of using Langevin dynamics with a very small damping constant, for example, $0.5 \mathrm{ps}^{-1}$, may be a reasonable compromise. A similar recommendation was made on the basis of a thorough analysis of different thermostats, although caution was still advised when using Langevin dynamics and related approaches. ${ }^{(108)}$

We also investigated another common approach used to speed up simulations, reducing the Lennard-Jones potential cutoff. Although C36 is parametrized for membranes based on a $12 \AA$ cutoff with a force-based switching function starting at $8 \AA$ (although typically set to $10 \AA$ to match the protein force field), ${ }^{(5)}$ a number of studies have pushed the cutoff to $9 \AA$ with no switching function. ${ }^{(37-39)}$ However, our results here show that there are trade-offs involved with this approximation, such as increased APL, disorder, and rate of diffusion (Figure 2). While these altered properties do not necessarily invalidate a simulation's results, their effects should be carefully considered. Looking ahead, approaches such as Lennard-Jones PME ${ }^{(109,110)}$ which obviates the need for a cutoff, are likely to overcome the problems noted here. ${ }^{(11)}$

\section{Supporting Information}

The Supporting Information is available free of charge on the ACS Publications website at DOI: $\underline{10.1021 / \text { acs.jctc.9b00160. }}$.

\section{Author Contributions}

${ }^{\#}$ C.B. and H.H. contributed equally to this work.

\section{Funding}

J.C.G. acknowledges support from the National Science Foundation (MCB-1452464) and the National Institutes of Health (R01-GM123169). Y.W. acknowledges Project 14323816 from the Research Grants Council as well as direct grant support from the Chinese University of Hong Kong. P.H.R. acknowledges support from the National Institute on Drug Abuse (RO1DA003934). Computational resources were provided through the Extreme Science and Engineering Discovery Environment (XSEDE), which is supported by NSF Grant OCI-1053575.

\section{Notes}

The authors declare no competing financial interest.

\section{References}

1. Lombard, J. Once upon a time the cell membranes: 175 years of cell boundary research. Biol. Direct. 2014, 9, 32, DOI: 10.1186/s13062-014-0032-7

2. Kučerka, N.; van Oosten, B.; Pan, J.; Heberle, F. A.; Harroun, T. A.; Katsaras, J. Molecular Structures of Fluid Phosphatidylethanolamine Bilayers Obtained from Simulation-toExperiment Comparisons and Experimental Scattering Density Profiles. J. Phys. Chem. $B$ 2015, 119, 1947- 1956, DOI: 10.1021/jp511159q 
3. Zhuang, X.; Makover, J. R.; Im, W.; Klauda, J. B. A systematic molecular dynamics simulation study of temperature dependent bilayer structural properties. Biochim. Biophys. Acta, Biomembr. 2014, 1838, 2520-2529, DOI: 10.1016/j.bbamem.2014.06.010

4. Botan, A.; Favela-Rosales, F.; Fuchs, P. F. J.; Javanainen, M.; Kanduč, M.; Kulig, W.; Lamberg, A.; Loison, C.; Lyubartsev, A.; Miettinen, M. S.; Monticelli, L.; Määttä, J.; Ollila, O. H. S.; Retegan, M.; Róg, T.; Santuz, H.; Tynkkynen, J. Toward Atomistic Resolution Structure of Phosphatidylcholine Headgroup and Glycerol Backbone at Different Ambient Conditions. J. Phys. Chem. B 2015, 119, 15075-15088, DOI: 10.1021/acs.jpcb.5b04878

5. Venable, R. M.; Brown, F. L. H.; Pastor, R. W. Mechanical properties of lipid bilayers from molecular dynamics simulation. Chem. Phys. Lipids 2015, 192, 60- 74, DOI: 10.1016/j.chemphyslip.2015.07.014

6. Zhuang, X.; Dávila-Contreras, E. M.; Beaven, A. H.; Im, W.; Klauda, J. B. An extensive simulation study of lipid bilayer properties with different head groups, acyl chain lengths, and chain saturations. Biochim. Biophys. Acta, Biomembr. 2016, 1858, 3093-3104, DOI: 10.1016/j.bbamem.2016.09.016

7. Pluhackova, K.; Kirsch, S. A.; Han, J.; Sun, L.; Jiang, Z.; Unruh, T.; Böckmann, R. A. A Critical Comparison of Biomembrane Force Fields: Structure and Dynamics of Model DMPC, POPC, and POPE Bilayers. J. Phys. Chem. B 2016, 120, 3888-3903, DOI: 10.1021/acs.jpcb.6b01870

8. Doktorova, M.; Harries, D.; Khelashvili, G. Determination of bending rigidity and tilt modulus of lipid membranes from real-space fluctuation analysis of molecular dynamics simulations. Phys. Chem. Chem. Phys. 2017, 19, 16806-16818, DOI: 10.1039/C7CP01921A

9. Wang, Y.; Zhao, T.; Wei, D.; Strandberg, E.; Ulrich, A. S.; Ulmschneider, J. P. How reliable are molecular dynamics simulations of membrane active antimicrobial peptides?. Biochim. Biophys. Acta, Biomembr. 2014, 1838, 2280- 2288, DOI: 10.1016/j.bbamem.2014.04.009

10. Lee, C. T.; Comer, J.; Herndon, C.; Leung, N.; Pavlova, A.; Swift, R. V.; Tung, C.; Rowley, C. N.; Amaro, R. E.; Chipot, C.; Wang, Y.; Gumbart, J. C. Simulation-Based Approaches for Determining Membrane Permeability of Small Compounds. J. Chem. Inf. Model. 2016, 56, 721- 733, DOI: 10.1021/acs.jcim.6b00022

11. Lyubartsev, A. P.; Rabinovich, A. L. Force Field Development for Lipid Membrane Simulations. Biochim. Biophys. Acta, Biomembr. 2016, 1858, 2483-2497, DOI: 10.1016/j.bbamem.2015.12.033

12. Poger, D.; Caron, B.; Mark, A. E. Validating lipid force fields against experimental data: Progress, challenges and perspectives. Biochim. Biophys. Acta, Biomembr. 2016, 1858, 1556- 1565, DOI: 10.1016/j.bbamem.2016.01.029 
13. Dickson, C. J.; Madej, B. D.; Skjevik, Å. A.; Betz, R. M.; Teigen, K.; Gould, I. R.; Walker, R. C. Lipid14: The AMBER Lipid Force Field. J. Chem. Theory

Comput. 2014, 10, 865-879, DOI: 10.1021/ct4010307

14. Jambeck, J. P. M.; Lyubartsev, A. P. An Extension and Further Validation of an AllAtomistic Force Field for Biological Membranes. J. Chem. Theory Comput. 2012, 8, 2938-2948, DOI: 10.1021/ct300342n

15. Klauda, J. B.; Venable, R. M.; Freites, J. A.; O’Connor, J. W.; Tobias, D. J.; MondragonRamirez, C.; Vorobyov, I.; MacKerell, A. D.; Pastor, R. W. Update of the CHARMM AllAtom Additive Force Field for Lipids: Validation on Six Lipid Types. J. Phys. Chem. $B$ 2010, 114, 7830- 7843, DOI: $10.1021 / j p 101759 q$

16. Kukol, A. Lipid Models for United-Atom Molecular Dynamics Simulations of Proteins. $J$. Chem. Theory Comput. 2009, 5, 615- 626, DOI: 10.1021/ct8003468

17. Schmid, N.; Eichenberger, A. P.; Choutko, A.; Riniker, S.; Winger, M.; Mark, A. E.; van Gunsteren, W. F. Definition and testing of the GROMOS force-field versions 54A7 and 54B7. Eur. Biophys. J. 2011, 40, 843- 856, DOI: 10.1007/s00249-011-0700-9

18. Poger, D.; Mark, A. E. Lipid Bilayers: The Effect of Force Field on Ordering and Dynamics. J. Chem. Theory Comput. 2012, 8, 4807-4817, DOI: 10.1021/ct300675z

19. Vanommeslaeghe, K.; Hatcher, E.; Acharya, C.; Kundu, S.; Zhong, S.; Shim, J.; Darian, E.; Guvench, O.; Lopes, P.; Vorobyov, I.; MacKerell, A. D., Jr. CHARMM General Force Field: A Force Field for Drug-Like Molecules Compatible with the CHARMM All-Atom Additive Biological Force Fields. J. Comput. Chem. 2009, 31, 671- 690, DOI: $10.1002 /$ jcc. 21367

20. Best, R. B.; Zhu, X.; Shim, J.; Lopes, P. E.; Mittal, J.; Feig, M.; MacKerell, A. D., Jr. Optimization of the additive CHARMM all-atom protein force field targeting improved sampling of the backbone $\phi, \psi$ and side-chain $\chi_{1}$ and $\mathrm{d} \chi_{2}$ ihedral angles. J. Chem. Theory Comput. 2012, 8, 3257-3273, DOI: 10.1021/ct300400x

21. Jo, S.; Kim, T.; Iyer, V. G.; Im, W. CHARMM-GUI: A web-based graphical user interface for CHARMM. J. Comput. Chem. 2008, 29, 1859- 1865, DOI: 10.1002/jcc.20945

22. Jo, S.; Lim, J. B.; Klauda, J. B.; Im, W. CHARMM-GUI Membrane Builder for Mixed Bilayers and Its Application to Yeast Membranes. Biophys. J. 2009, 97, 50- 58, DOI: 10.1016/j.bpj.2009.04.013

23. Wu, E. L.; Cheng, X.; Jo, S.; Rui, H.; Song, K. C.; Dávila-Contreras, E. M.; Qi, Y.; Lee, J.; Monje-Galvan, V.; Venable, R. M.; Klauda, J. B.; Im, W. CHARMM-GUI Membrane Builder toward realistic biological membrane simulations. J. Comput.

Chem. 2014, 35, 1997- 2004, DOI: 10.1002/jcc.23702 
24. Lee, J.; Cheng, X.; Swails, J. M.; Yeom, M. S.; Eastman, P. K.; Lemkul, J. A.; Wei, S.; Buckner, J.; Jeong, J. C.; Qi, Y.; Jo, S.; Pande, V. S.; Case, D. A.; Brooks, C.

L.; MacKerell, A. D.; Klauda, J. B.; Im, W. CHARMM-GUI Input Generator for NAMD, GROMACS, AMBER, OpenMM, and CHARMM/OpenMM Simulations Using the CHARMM36 Additive Force Field. J. Chem. Theory Comput. 2016, 12, 405-413, DOI: 10.1021/acs.jctc.5b00935

25. Wang, Y.; Markwick, P. R. L.; de Oliveira, C. A. F.; McCammon, J. A. Enhanced Lipid Diffusion and Mixing in Accelerated Molecular Dynamics. J. Chem. Theory Comput. 2011, 7, 3199-3207, DOI: 10.1021/ct200430c

26. Feenstra, K. A.; Hess, B.; Berendsen, H. J. C. Improving efficiency of large time-scale molecular dynamics simulations of hydrogen-rich systems. J. Comput. Chem. 1999, 20, 786- 798, DOI: 10.1002/(SICI)1096-987X(199906)20:8<786::AIDJCC5>3.0.CO;2-B

27. Hopkins, C. W.; Le Grand, S.; Walker, R. C.; Roitberg, A. E. Long-Time-Step Molecular Dynamics through Hydrogen Mass Repartitioning. J. Chem. Theory

Comput. 2015, 11, 1864-1874, DOI: 10.1021/ct5010406

28. Francesco, R.; Martin, S. Thermodynamics and kinetics of large-time-step molecular dynamics. J. Comput. Chem. 2012, 33, 475-483, DOI: 10.1002/jcc.21990

29. Ryckaert, J.-P.; Ciccotti, G.; Berendsen, H. J. C. Numerical integration of the Cartesian equations of motion of a system with constraints: molecular dynamics of n-alkanes. $J$. Comput. Phys. 1977, 23, 327-341, DOI: 10.1016/0021-9991(77)90098-5

30. Miyamoto, S.; Kollman, P. A. Settle: An analytical version of the SHAKE and RATTLE algorithm for rigid water models. J. Comput. Chem. 1992, 13, 952-962, DOI: $10.1002 /$ jcc. 540130805

31. Loubet, B.; Kopec, W.; Khandelia, H. Accelerating All-Atom MD Simulations of Lipids Using a Modified Virtual-Sites Technique. J. Chem. Theory

Comput. 2014, 10, 5690- 5695, DOI: 10.1021/ct500100f

32. Bjelkmar, P.; Larsson, P.; Cuendet, M. A.; Hess, B.; Lindahl, E. Implementation of the CHARMM Force Field in GROMACS: Analysis of Protein Stability Effects from Correction Maps, Virtual Interaction Sites, and Water Models. J. Chem. Theory

Comput. 2010, 6, 459-466, DOI: 10.1021/ct900549r

33. Olesen, K.; Awasthi, N.; Bruhn, D. S.; Pezeshkian, W.; Khandelia, H. Faster Simulations with a $5 \mathrm{fs}$ Time Step for Lipids in the CHARMM Force Field. J. Chem. Theory Comput. 2018, 14, 3342-3350, DOI: 10.1021/acs.jctc.8b00267 
34. Phillips, J. C.; Braun, R.; Wang, W.; Gumbart, J.; Tajkhorshid, E.; Villa, E.; Chipot, C.; Skeel, R. D.; Kale, L.; Schulten, K. Scalable molecular dynamics with NAMD. J. Comput. Chem. 2005, 26, 1781-1802, DOI: 10.1002/jcc.20289

35. Case, D.; Betz, R.; Cerutti, D.; Cheatham, T., III; Darden, T.; Duke, R.; Giese, T.; Gohlke, H.; Goetz, A.; Homeyer, N.; Izadi, S.; Janowski, P.; Kaus, J.; Kovalenko, A.; Lee, T.; LeGrand, S.; Li, P.; Lin, C.; Luchko, T.; Luo, R.; Madej, B.; Mermelstein, D.; Merz, K.; Monard, G.; Nguyen, H.; Nguyen, H.; Omelyan, I.; Onufriev, A.; Roe, D. R.; Roitberg, A.; Sagui, C.; Simmerling, C.; Botello-Smith, W.; Swails, J.; Walker, R.; Wang, J.; Wolf, R.; Wu, X.; Xiao, L.; Kollman, P. AMBER 2016; University of California: San Francisco, CA, 2016.

36. Harvey, M. J.; Giupponi, G.; Fabritiis, G. D. ACEMD: Accelerating Biomolecular Dynamics in the Microsecond Time Scale. J. Chem. Theory Comput. 2009, 5, 1632- 1639, DOI: $10.1021 /$ ct9000685

37. Petruk, A. A.; Varriale, S.; Coscia, M. R.; Mazzarella, L.; Merlino, A.; Oreste, U. The structure of the CD3 $\zeta \zeta$ transmembrane dimer in POPC and raft-like lipid bilayer: A molecular dynamics study. Biochim. Biophys. Acta,

Biomembr. 2013, 1828, 2637-2645, DOI: 10.1016/j.bbamem.2013.07.019

38. Liu, X.; Ahn, S.; Kahsai, A. W.; Meng, K.-C.; Latorraca, N. R.; Pani, B.; Venkatakrishnan, A. J.; Masoudi, A.; Weis, W. I.; Dror, R. O.; Chen, X.; Lefkowitz, R. J.; Kobilka, B. K. Mechanism of intracellular allosteric $\beta_{2}$ AR antagonist revealed by X-ray crystal structure. Nature 2017, 548, 480-484, DOI: 10.1038/nature23652

39. Wacker, D.; Wang, S.; McCorvy, J. D.; Betz, R. M.; Venkatakrishnan, A. J.; Levit, A.; Lansu, K.; Schools, Z. L.; Che, T.; Nichols, D. E.; Shoichet, B. K.; Dror, R. O.; Roth, B. L. Crystal Structure of an LSD-Bound Human Serotonin Receptor. Cell 2017, 168, 377-389.e12, DOI: 10.1016/j.cell.2016.12.033

40. Pandit, K. R.; Klauda, J. B. Membrane models of E. coli containing cyclic moieties in the aliphatic lipid chain. Biochim. Biophys. Acta, Biomembr. 2012, 1818, 1205-1210, DOI: 10.1016/j.bbamem.2012.01.009

41. Braun, A. R.; Brandt, E. G.; Edholm, O.; Nagle, J. F.; Sachs, J. N. Determination of Electron Density Profiles and Area from Simulations of Undulating Membranes. Biophys. J. 2011, 100, 2112-2120, DOI: 10.1016/j.bpj.2011.03.009

42. Hong, C.; Tieleman, D. P.; Wang, Y. Microsecond molecular dynamics simulations of lipid mixing. Langmuir 2014, 30, 11993- 12001, DOI: 10.1021/1a502363b

43. Michaud-Agrawal, N.; Denning, E. J.; Woolf, T. B.; Beckstein, O. MDAnalysis: a toolkit for the analysis of molecular dynamics simulations. J. Comput.

Chem. 2011, 32, 2319-2327, DOI: 10.1002/jcc.21787 
44. Grossfield, A.; Zuckerman, D. M. Quantifying uncertainty and sampling quality in biomolecular simulations. Annu. Rep. Comput. Chem. 2009, 5, 23-48, DOI: 10.1016/S15741400(09)00502-7

45. Yamashita, E.; Zhalnina, M. V.; Zakharov, S. D.; Sharma, O.; Cramer, W. A. Crystal structures of the OmpF porin: function in a colicin translocon. $E M B O$ J. 2008, 27, 2171-2180, DOI: 10.1038/emboj.2008.137

46. Pezeshki, S.; Chimerel, C.; Bessonov, A. N.; Winterhalter, M.; Kleinekathofer, U. Understanding ion conductance on a molecular level: an all-atom modeling of the bacterial porin OmpF. Biophys. J. 2009, 97, 1898-1906, DOI: 10.1016/j.bpj.2009.07.018

47. Crozier, P. S.; Henderson, D.; Rowley, R. L.; Busath, D. D. Model channel ion currents in $\mathrm{NaCl}$-extended simple point charge water solution with applied-field molecular dynamics. Biophys. J. 2001, 81, 3077-3089, DOI: 10.1016/S0006-3495(01)75946-2

48. Aksimentiev, A.; Schulten, K. Imaging alpha-hemolysin with molecular dynamics: ionic conductance, osmotic permeability, and the electrostatic potential map. Biophys. J. 2005, 88, 3745- 3761, DOI: 10.1529/biophysj.104.058727

49. Hurst, D. P.; Grossfield, A.; Lynch, D. L.; Feller, S.; Romo, T. D.; Gawrisch, K.; Pitman, M. C.; Reggio, P. H. A Lipid Pathway for Ligand Binding Is Necessary for a Cannabinoid G Protein-coupled Receptor. J. Biol. Chem. 2010, 285, 17954- 17964, DOI: 10.1074/jbc.M109.041590

50. Romo, T.; Grossfield, A. LOOS: An extensible platform for the structural analysis of simulations. 31st Annual International Conference of the IEEE EMBS 2009, 2332-2335, DOI: 10.1109/IEMBS.2009.5335065

51. Romo, T. D.; Leioatts, N.; Grossfield, A. Lightweight object oriented structure analysis: Tools for building tools to analyze molecular dynamics simulations. J. Comput. Chem. 2014, 35, 2305-2318, DOI: 10.1002/jcc.23753

52. Rapaport, D. Hydrogen bonds in water. Network organization and lifetimes. Mol. Phys. 1983, 50, 1151- 1162, DOI: 10.1080/00268978300102931

53. Gumbart, J. C.; Ulmschneider, M. B.; Hazel, A.; White, S. H.; Ulmschneider, J. P. Computed Free Energies of Peptide Insertion into Bilayers are Independent of Computational Method. J. Membr. Biol. 2018, 251, 345-356, DOI: 10.1007/s00232-018-0026-y

54. Ulmschneider, M. B.; Doux, J. P.; Killian, J. A.; Smith, J. C.; Ulmschneider, J. P. Mechanism and kinetics of peptide partitioning into membranes from all-atom simulations of thermostable peptides. J. Am. Chem. Soc. 2010, 132, 3452-3460, DOI: 10.1021/ja909347x 
55. Domański, J.; Sansom, M. S. P.; Stansfeld, P. J.; Best, R. B. Balancing force field proteinlipid interactions to capture transmembrane helix-helix association. J. Chem. Theory Comput. 2018, 14, 1706-1715, DOI: 10.1021/acs.jctc.7b00983

56. Humphrey, W.; Dalke, A.; Schulten, K. VMD - Visual Molecular Dynamics. J. Mol. Graphics 1996, 14, 33-38, DOI: 10.1016/0263-7855(96)00018-5

57. Darden, T. A.; York, D. M.; Pedersen, L. G. Particle mesh Ewald: An $N \log N$ method for Ewald sums in large systems. J. Chem. Phys. 1993, 98, 10089-10092, DOI: $10.1063 / 1.464397$

58. Feller, S. E.; Zhang, Y. H.; Pastor, R. W.; Brooks, B. R. Constant pressure molecular dynamics simulations - The Langevin piston method. J. Chem.

Phys. 1995, 103, 4613-4621, DOI: 10.1063/1.470648

59. Jorgensen, W. L.; Chandrasekhar, J.; Madura, J. D.; Impey, R. W.; Klein, M. L. Comparison of simple potential functions for simulating liquid water. J. Chem.

Phys. 1983, 79, 926- 935, DOI: 10.1063/1.445869

60. Huang, J.; Rauscher, S.; Nawrocki, G.; Ran, T.; Feig, M.; de Groot, B. L.; Grubmüller, H.; MacKerell, A. D., Jr CHARMM36m: an improved force field for folded and intrinsically disordered proteins. Nat. Methods 2017, 14, 71-73, DOI: 10.1038/nmeth.4067

61. Grossfield, A. WHAM: The Weighted Histogram Analysis Method, version 2.0.6, 2012; URL: http://membrane.urmc.rochester.edu/content/wham.

62. Nagle, J. F.; Tristram-Nagle, S. Structure of lipid bilayers. Biochim. Biophys. Acta, Rev. Biomembr. 2000, 1469, 159- 195, DOI: 10.1016/S0304-4157(00)00016-2

63. Benz, R. W.; Castro-Román, F.; Tobias, D. J.; White, S. H. Experimental Validation of Molecular Dynamics Simulations of Lipid Bilayers: A New Approach. Biophys. J. 2005, 88, 805- 817, DOI: 10.1529/biophysj.104.046821

64. Petrache, H. I.; Dodd, S. W.; Brown, M. F. Area per lipid and acyl length distributions in fluid phosphatidylcholines determined by $2 \mathrm{H}$ NMR spectroscopy. Biophys.

J. 2000, 79, 3172-3192, DOI: 10.1016/S0006-3495(00)76551-9

65. Kučerka, N.; Tristram-Nagle, S.; Nagle, J. F. Closer look at structure of fully hydrated fluid phase DPPC bilayers. Biophys. J. 2006, 90, L83- L85, DOI: 10.1529/biophysj.106.086017

66. Kučerka, N.; Nieh, M. P.; Katsaras, J. Fluid phase lipid areas and bilayer thicknesses of commonly used phosphatidylcholines as a function of temperature. Biochim. Biophys. Acta, Biomembr. 2011, 1808, 2761-2771, DOI: 10.1016/j.bbamem.2011.07.022 
67. Jung, J.; Kobayashi, C.; Sugita, Y. Optimal Temperature Evaluation in Molecular Dynamics Simulations with a Large Time Step. J. Chem. Theory Comput. 2019, 15, 84- 94, DOI: 10.1021/acs.jctc. 8 b00874

68. Kučerka, N.; Nagle, J. F.; Sachs, J. N.; Feller, S. E.; Pencer, J.; Jackson, A.; Katsaras, J. Lipid Bilayer Structure Determined by the Simultaneous Analysis of Neutron and X-Ray Scattering Data. Biophys. J. 2008, 95, 2356-2367, DOI: 10.1529/biophysj.108.132662

69. Orädd, G.; Westerman, P. W.; Lindblom, G. Lateral diffusion coefficients of separate lipid species in a ternary raft-forming bilayer: a pfg-NMR multinuclear study. Biophys. J. 2005, 89, 315- 320, DOI: 10.1529/biophysj.105.061762

70. Rappolt, M.; Hickel, A.; Bringezu, F.; Lohner, K. A. Mechanism of the lamellar/inverse hexagonal phase transition examined by high resolution X-ray diffraction. Biophys. J. 2003, 84, 3111-3122, DOI: 10.1016/S0006-3495(03)70036-8

71. Rand, R. P.; Parsegian, V. A. Hydration forces between phospholipid bilayers. Biochim. Biophys. Acta, Rev. Biomembr. 1989, 988, 351-376, DOI: 10.1016/0304-4157(89)90010-5

72. Kučerka, N.; Tristram-Nagle, S.; Nagle, J. F. Structure of Fully Hydrated Fluid Phase Lipid Bilayers with Monounsaturated Chains. J. Membr. Biol. 2006, 208, 193- 202, DOI: 10.1007/s00232-005-7006-8

73. Evans, E.; Rawicz, W.; Smith, B. A. Back to the future: mechanics and thermodynamics of lipid biomembranes. Faraday Discuss. 2013, 161, 591- 611, DOI: 10.1039/C2FD20127E

74. Heinemann, F.; Betaneli, V.; Thomas, F. A.; Schwille, P. Quantifying Lipid Lateral Diffusion by Fluorescence Correlation Spectroscopy: A Critical Treatise. Langmuir 2012, 28, 13395- 13404, DOI: 10.1021/la302596h

75. Hwang, H.; Paracini, N.; Parks, J. M.; Lakey, J. H.; Gumbart, J. C. Distribution of mechanical stress in the Escherichia coli cell envelope. Biochim. Biophys. Acta, Biomembr. 2018, 1860, 2566- 2575, DOI: 10.1016/j.bbamem.2018.09.020

76. Egberts, E.; Marrink, S.; Berendsen, H. J. C. Molecular dynamics simulation of a phospholipid membrane. Eur. Biophys. J. 1994, 22, 423-436, DOI: 10.1007/BF00180163

77. Wohlert, J.; Edholm, O. Dynamics in atomistic simulations of phospholipid membranes: Nuclear magnetic resonance relaxation rates and lateral diffusion. J. Chem. Phys. 2006, 125, 204703-1- 10, DOI: 10.1063/1.2393240

78. Vermeer, L. S.; de Groot, B. L.; Réat, V.; Milon, A.; Czaplicki, J. Acyl chain order parameter profiles in phospholipid bilayers: Computation from molecular dynamics simulations and comparison with ${ }^{2} \mathrm{H}$ NMR experiments. Eur. Biophys. J. 2007, 36, 919- 931, DOI: $10.1007 / \mathrm{s} 00249-007-0192-9$ 
79. Camley, M.; Lerner, B. A.; Pastor, R.; Brown, F. Strong influence of periodic boundary conditions on lateral diffusion in lipid bilayer membranes. J. Chem.

Phys. 2015, 143, 243113, DOI: 10.1063/1.4932980

80. Venable, R. M.; Ingolfsson, H. I.; Lerner, M. G.; Perrin, B. S.; Camley, B. A.; Marrink, S. J.; Brown, F. L. H.; Pastor, R. W. Lipid and Peptide Diffusion in Bilayers: The SaffmanDelbrück Model and Periodic Boundary Conditions. J. Phys. Chem.

B 2017, 121, 3443-3457, DOI: 10.1021/acs.jpcb.6b09111

81. Lindblom, G.; Oradd, G. Lipid lateral diffusion and membrane heterogeneity. Biochim. Biophys. Acta, Biomembr. 2009, 1788, 234-244, DOI: 10.1016/j.bbamem.2008.08.016

82. Koopman, E. A.; Lowe, C. P. Advantages of a Lowe-Andersen thermostat in molecular dynamics simulations. J. Chem. Phys. 2006, 124, 204103, DOI: 10.1063/1.2198824

83. Mishra, B.; Schlick, T. The notion of error in Langevin dynamics. I. Linear analysis. $J$. Chem. Phys. 1996, 105, 299-318, DOI: 10.1063/1.471875

84. Fincham, D. Choice of timestep in molecular dynamics simulation. Comput. Phys. Commun. 1986, 40, 263- 269, DOI: 10.1016/0010-4655(86)90113-X

85. Shinoda, W.; Namiki, N.; Okazaki, S. Molecular dynamics study of a lipid bilayer: Convergence, structure, and long-time dynamics. J. Chem.

Phys. 1997, 106, 5731- 5743, DOI: 10.1063/1.473592

86. Bennett, W. D.; Hong, C. K.; Wang, Y.; Tieleman, D. P. Antimicrobial Peptide Simulations and the Influence of Force Field on the Free Energy for Pore Formation in Lipid Bilayers. $J$. Chem. Theory Comput. 2016, 12, 4524- 4533, DOI: 10.1021/acs.jctc.6b00265

87. Brandt, E. G.; Braun, A. R.; Sachs, J. N.; Nagle, J. F.; Edholm, O. Interpretation of fluctuation spectra in lipid bilayer simulations. Biophys. J. 2011, 100, 2104-2111, DOI: 10.1016/j.bpj.2011.03.010

88. Braun, A. R.; Brandt, E. G.; Edholm, O.; Nagle, J. F.; Sachs, J. N. Determination of electron density profiles and area from simulations of undulating membranes. Biophys.

J. 2011, 100, 2112-2120, DOI: 10.1016/j.bpj.2011.03.009

89. Marsh, D. Elastic curvature constants of lipid monolayers and bilayers. Chem. Phys. Lipids 2006, 144, 146- 159, DOI: 10.1016/j.chemphyslip.2006.08.004

90. Cowan, S. W.; Garavito, R. M.; Jansonius, J. N.; Jenkins, J. A.; Karlsson, R.; Konig, N.; Pai, E. F.; Pauptit, R. A.; Rizkallah, P. J.; Rosenbusch, J. P. The structure of OmpF porin in a tetragonal crystal form. Structure 1995, 3, 1041-1050, DOI: 10.1016/S09692126(01)00240-4 
91. Watanabe, M.; Rosenbusch, J.; Schirmer, T.; Karplus, M. Computer simulations of the OmpF porin from the outer membrane of Escherichia coli. Biophys. J. 1997, 72, 2094-2102, DOI: 10.1016/S0006-3495(97)78852-0

92. Latorraca, N. R.; Venkatakrishnan, A. J.; Dror, R. O. GPCR Dynamics: Structures in Motion. Chem. Rev. 2017, 117, 139-155, DOI: 10.1021/acs.chemrev.6b00177

93. Marino, K. A.; Prada-Gracia, D.; Provasi, D.; Filizola, M. Impact of Lipid Composition and Receptor Conformation on the Spatio-temporal Organization of $\mu$-Opioid Receptors in a Multi-component Plasma Membrane Model. PLoS Comput. Biol. 2016, 12, e1005240 DOI: 10.1371/journal.pcbi. 1005240

94. Grossfield, A.; Feller, S. E.; Pitman, M. C. Convergence of Molecular Dynamics Simulations of Membrane Proteins. Proteins: Struct., Funct., Genet. 2007, 67, 31-40, DOI: $10.1002 /$ prot. 21308

95. Romo, T. D.; Grossfield, A. Block Covariance Overlap Method and Convergence in Molecular Dynamics Simulations. J. Chem. Theory Comput. 2011, 7, 2464- 2472, DOI: $10.1021 /$ ct2002754

96. Sotudeh, N.; Morales, P.; Hurst, D. P.; Lynch, D. L.; Reggio, P. H. Towards A Molecular Understanding of The Cannabinoid Related Orphan Receptor GPR18: A Focus on its Constitutive Activity. Int. J. Mol. Sci. 2019, 20, 2300, DOI: 10.3390/ijms20092300

97. Petrache, H. I.; Grossfield, A.; MacKenzie, K. R.; Engelman, D. M.; Woolf, T. B. Modulation of glycophorin A transmembrane helix interactions by lipid bilayers: molecular dynamics calculations. J. Mol. Biol. 2000, 302, 727- 746, DOI: 10.1006/jmbi.2000.4072

98. Henin, J.; Pohorille, A.; Chipot, C. Insights into the recognition and association of transmembrane alpha-helices. The free energy of alpha-helix dimerization in glycophorin A. J. Am. Chem. Soc. 2005, 127, 8478-8484, DOI: $10.1021 /$ ja050581y

99. Cuthbertson, J. M.; Bond, P. J.; Sansom, M. S. Transmembrane helix-helix interactions: comparative simulations of the glycophorin a dimer. Biochemistry 2006, 45, 14298-14310, DOI: 10.1021/bi0610911

100. Zhang, J.; Lazaridis, T. Calculating the free energy of association of transmembrane helices. Biophys. J. 2006, 91, 1710- 1723, DOI: 10.1529/biophysj.106.081224

101. Lee, J.; Im, W. Role of hydrogen bonding and helix-lipid interactions in transmembrane helix association. J. Am. Chem. Soc. 2008, 130, 6456- 6462, DOI: 10.1021/ja711239h

102. Psachoulia, E.; Marshall, D. P.; Sansom, M. S. Molecular dynamics simulations of the dimerization of transmembrane alpha-helices. Acc. Chem. Res. 2010, 43, 388- 396, DOI: 10.1021/ar900211k 
103. Janosi, L.; Prakash, A.; Doxastakis, M. Lipid-modulated sequence-specific association of glycophorin A in membranes. Biophys. J. 2010, 99, 284- 292, DOI:

10.1016/j.bpj.2010.04.005

104. Sengupta, D.; Marrink, S. J. Lipid-mediated interactions tune the association of glycophorin A helix and its disruptive mutants in membranes. Phys. Chem. Chem.

Phys. 2010, 12, 12987- 12996, DOI: 10.1039/c0cp00101e

105. Li, P. C.; Miyashita, N.; Im, W.; Ishido, S.; Sugita, Y. Multidimensional umbrella sampling and replica-exchange molecular dynamics simulations for structure prediction of transmembrane helix dimers. J. Comput. Chem. 2014, 35, 300- 308, DOI:

$10.1002 /$ jcc. 23494

106. Mori, T.; Miyashita, N.; Im, W.; Feig, M.; Sugita, Y. Molecular dynamics simulations of biological membranes and membrane proteins using enhanced conformational sampling algorithms. Biochim. Biophys. Acta, Biomembr. 2016, 1858, 1635-1651, DOI: 10.1016/j.bbamem.2015.12.032

107. Domański, J.; Hedger, G.; Best, R. B.; Stansfeld, P. J.; Sansom, M. S. P. Convergence and Sampling in Determining Free Energy Landscapes for Membrane Protein Association. $J$. Phys. Chem. B 2017, 121, 3364- 3375, DOI: 10.1021/acs.jpcb.6b08445

108. Basconi, J. E.; Shirts, M. R. Effects of temperature control algorithms on transport properties and kinetics in molecular dynamics simulations. J. Chem. Theory

Comput. 2013, 9, 2887-2899, DOI: 10.1021/ct400109a

109. Wennberg, C. L.; Murtola, T.; Hess, B.; Lindahl, E. Lennard-Jones Lattice Summation in Bilayer Simulations Has Critical Effects on Surface Tension and Lipid Properties. J. Chem. Theory Comput. 2013, 9, 3527- 3537, DOI: 10.1021/ct400140n

110. Leonard, A. N.; Pastor, R. W.; Klauda, J. B. Parameterization of the CHARMM AllAtom Force Field for Ether Lipids and Model Linear Ethers. J. Phys. Chem.

B 2018, 122, 6744- 6754, DOI: 10.1021/acs.jpcb.8b02743

111. Leonard, A. N.; Simmonett, A. C.; Pickard IV, F. C.; Huang, J.; Venable, R. M.; Klauda, J. B.; Brooks, B. R.; Pastor, R. W. Comparison of Additive and Polarizable Models with Explicit Treatment of Long-Range Lennard-Jones Interactions Using Alkane Simulations. $J$. Chem. Theory Comput. 2018, 14, 948- 958, DOI: 10.1021/acs.jctc.7b00948 